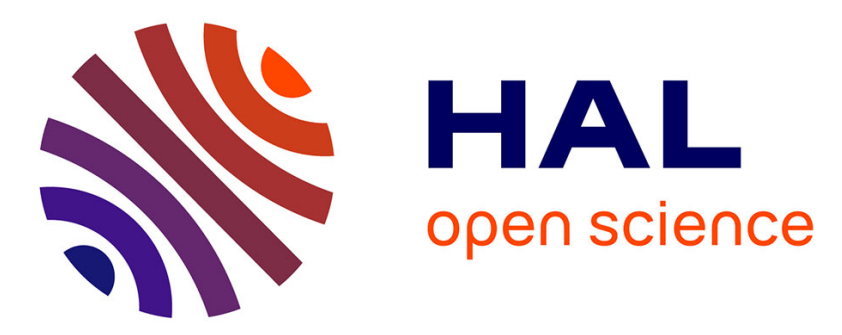

\title{
Vibrational cooling in the liquid phase studied by ultrafast investigations of cycloheptatriene
}

Oliver Schalk, Ji-Ping Yang, Andreas Hertwig, Horst Hippler, Andreas Neil Unterreiner

\section{- To cite this version:}

Oliver Schalk, Ji-Ping Yang, Andreas Hertwig, Horst Hippler, Andreas Neil Unterreiner. Vibrational cooling in the liquid phase studied by ultrafast investigations of cycloheptatriene. Molecular Physics, 2009, 107 (20), pp.2159-2167. 10.1080/00268970903193010 . hal-00521567

\section{HAL Id: hal-00521567 https://hal.science/hal-00521567}

Submitted on 28 Sep 2010

HAL is a multi-disciplinary open access archive for the deposit and dissemination of scientific research documents, whether they are published or not. The documents may come from teaching and research institutions in France or abroad, or from public or private research centers.
L'archive ouverte pluridisciplinaire HAL, est destinée au dépôt et à la diffusion de documents scientifiques de niveau recherche, publiés ou non, émanant des établissements d'enseignement et de recherche français ou étrangers, des laboratoires publics ou privés. 


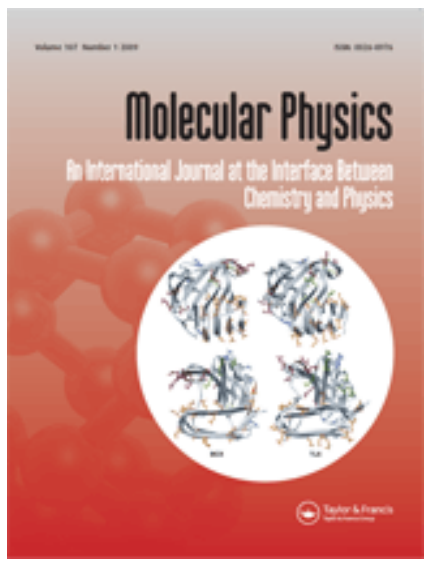

\section{Vibrational cooling in the liquid phase studied by ultrafast investigations of cycloheptatriene}

\begin{tabular}{|c|c|}
\hline Journal: & Molecular Physics \\
\hline Manuscript ID: & TMPH-2009-0160.R1 \\
\hline Manuscript Type: & Full Paper \\
\hline $\begin{array}{r}\text { Date Submitted by the } \\
\text { Author: }\end{array}$ & 01-Jul-2009 \\
\hline Complete List of Authors: & $\begin{array}{l}\text { Schalk, Oliver; Steacie Institute for Molecular Sciences } \\
\text { Yang, Ji-Ping; Department of Applied Physics and Institute of Laser } \\
\text { Technology } \\
\text { Hertwig, Andreas; BAM } \\
\text { Hippler, Horst; Physikalische Chemie } \\
\text { Unterreiner, Andreas; University of Karlsruhe, Physical Chemistry }\end{array}$ \\
\hline Keywords: & $\begin{array}{l}\text { vibrational cooling, ultrafast dynamics, cycloheptatriene, internal } \\
\text { conversion, Sulzer-Wieland model }\end{array}$ \\
\hline \multicolumn{2}{|c|}{$\begin{array}{l}\text { Note: The following files were submitted by the author for peer review, but cannot be converted } \\
\text { to PDF. You must view these files (e.g. movies) online. }\end{array}$} \\
\hline New WinZip File.zip & \\
\hline
\end{tabular}

\section{(s) ScholaroNE" \\ Manuscript Central}




\begin{abstract}
Solvent-mediated vibrational relaxation in UV pump-probe experiments has been studied using cycloheptatriene (CHT) and its perdeuterated counterpart (CHTd8) as model systems. Transient spectra containing absolute $\epsilon(t)$-values are obtained by a modified Sulzer-Wieland ansatz and a global fit function for vibrational relaxation is proposed.
\end{abstract}

\title{
1. Introduction
}

Ultrafast relaxation of vibrationally excited molecules in the electronic ground state can be observed in many ultrafast pump-probe studies if a molecule is excited to a higher lying electronic state and subsequently returning to the ground state by one (or more) internal conversions to highly excited vibrationally states. Often, the energy dissipation to the solvent is treated only qualitatively lacking a detailed knowledge of the time-dependent evolution of the relevant absorption coefficients. In addition, photoexcitation may lead to a non-thermal energy distribution on an ultrashort timescale. For a more adequate description, additional information is then necessary. Here, we demonstrate that 1,3,5cycloheptatriene (CHT) may be regarded as a model system towards a more quantitative assignment of transient spectra including time-dependent absorption coefficients.

During the last decade, CHT and related compounds have been studied extensively by various spectroscopic techniques from nanosecond to femtosecond (fs) timescale [1]-[10]. From these studies, the following picture emerges: After

\footnotetext{
${ }^{*}$ Corresponding author

Email address: andreas.unterreiner@kit.edu (Andreas N. Unterreiner)
} 
excitation around $260 \mathrm{~nm}$ to the $S_{2}$-state, the molecule passes two conical intersections $\left(S_{1}-S_{2}\right.$ and $\left.S_{0}-S_{1}\right)$ and reaches the ground state in $\tau_{I C}=(110 \pm 15)$ fs $[5,6,9]$ with a vibrational temperature of $2200 \mathrm{~K}$ [8]. Despite a typically quite narrow energy distribution that cannot be described canonically, it has been shown that the spectra of microcanonical and canonical distributions of the same temperature exhibit the same spectrum in second order perturbation theory [11]. Although there is some discrepancy in the literature concerning the timescale for the second intersection, a $[1,7]$-sigmatropic hydrogen migration is supposed to take place in the fs-regime as recently suggested by additional experiments on the perchlorinated counterpart of CHT [10]. Due to these fast internal conversions, CHT is ideally suited to study vibrational relaxation since no energy is dissipated to the surrounding solvent until reaching the electronic ground state. Necessary parameters for spectra reconstruction can be obtained from vibrational cooling studies after shock waves in the gaseous phase between 300 and $1200 \mathrm{~K}[12,11]$ and by pump-probe spectroscopy using 500 fs-pulses in compressed gases, supercritical fluids and liquids [13, 14]. The spectra obtained from shock wave experiments have been explained by means of a modified Sulzer-Wieland ansatz [15] while pump-probe experiments were used to extract the energy loss due to collisions in media of different densities.

In this paper, we show that the modified Sulzer-Wieland approach can be transfered to femtosecond time resolved measurements to construct transient spectra with quantitatively determined absorption coefficients $\epsilon(t)$ after excitation of CHT and present a method to fit the vibrational cooling observed in the transients of pump-probe experiments.

\section{Experimental methods}

\subsection{Sample preparation}

CHT was used as obtained from Fluka with a nominal purity of $>97 \%$ and $\mathrm{CHTd} 8$ has been synthesized by Dr. Eckert GmbH (Germany) according to the procedure described by Engdahl et al. [16]. The purities (about $90 \%$ for CHTd8) [9] have been checked by ${ }^{1} \mathrm{H}-\mathrm{NMR}$ and ${ }^{13} \mathrm{C}-\mathrm{NMR}$ in $\mathrm{CDCl}_{3}$ (Bruker, $250 \mathrm{MHz}$ ), UV-vis spectroscopy (Varian, Cary 5e) and GC-MS for CHTd8. The UV-vis spectra for CHT and CHTd8 are shown in Fig. 1. The spectrum of CHT is smooth and featureless as in essence holds for CHTd8, too. In addition, a small blue-shift of the absorption maximum of $3 \mathrm{~nm}$ compared to CHT is observed. A more detailed analysis showed that impurities in CHTd8 are mostly toluene and partially deuterated CHT (CHT-d6). Toluene gave a pump probe signal at $395 \mathrm{~nm}$ that was more than ten times smaller than for CHT and was therefore neglected. Also, no evidence of a contribution of partially deuterated CHT could be found.

\subsection{Experimental setup}

Two commercial laser systems with $1 \mathrm{kHz}$ repetition rate were used in this study: (i) a multi-pass amplifier system (FEMTOPRO, Femtolasers) with 30 fs 


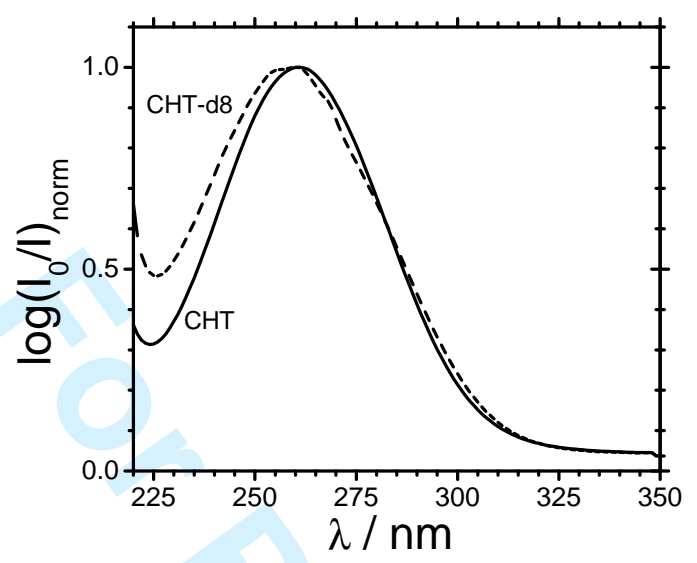

Figure 1: UV-Vis spectra of CHT and CHTd8 in cyclohexane, adopted from Ref. [9].

pulses at $790 \mathrm{~nm}[17,18]$ and (ii) a regenerative amplifier (CPA 2001, ClarkMXR) with $150 \mathrm{fs}$ pulses at $775 \mathrm{~nm}$.

The output of the multi-pass system was modified for pump probe spectroscopy by reducing the laser spot size by a factor of 3 to a diameter of 6 $\mathrm{mm}$ with two telescopes. Due to the high intensities the beam had to be split using a broad-band beam splitter and half of the output energy was used for further frequency conversion. The second harmonic was generated in a $100 \mu \mathrm{m}$ $\mathrm{BBO}$ crystal (type I) and separated by a dichroic mirror from the fundamental. By collinearly recombining the fundamental and the second harmonic light the third harmonic was generated in another $100 \mu \mathrm{m}$ BBO crystal (type I) and typically less than $2 \mu \mathrm{J}$ were used unfocused as pump pulse at $263 \mathrm{~nm}$. The 395 $\mathrm{nm}$ beam served as probe pulse after being attenuated to energies below $50 \mathrm{~nJ}$ by quartz plates. For a more detailed experimental setup see Ref. [19].

The regenerative amplifier served as a pump source for two independently tunable NOPA (noncollinear optical parametric amplifier) systems [20, 21]. The wavelength of one NOPA was set to $526 \mathrm{~nm}$ and frequency doubled to $263 \mathrm{~nm}$ with a typical energy of $0.5 \mu \mathrm{J}$ and served as pump pulse while the second NOPA was tuned between 298 and $373 \mathrm{~nm}$ at moderate energies below $10 \mathrm{~nJ}$. Pump and probe beams were directed separately onto two curved mirrors and focused into the sample. The polarization of the pulses were set parallel to avoid any additional dispersive elements in the beam path. Since it was shown that the anisotropy of these systems are nearly zero at delay times relevant for the the study of vibrational cooling ( $t \geq 500 \mathrm{fs}$ ) [9], no polarization effects need to be considered in the analysis.

Care was taken to ensure that the diameter of the pump pulse was always larger than the probe beam diameter. In both set-ups pump and probe beams intersected an angle of $4^{\circ}$ at the sample position. The pump beam was chopped 
at $500 \mathrm{~Hz}$ and temporally changed relative to the probe beam using a computer controlled translation stage (Melles Griot). Intensities of the probe pulse before and behind the cell were measured by photodiodes and the relative change of the optical density with and without the pump pulse, $\triangle \mathrm{OD}$, was recorded. To improve the signal-to-noise ratio time profiles were recorded by averaging 50 laser shots at each delay time for a single scan and 20 subsequent scans were averaged. The experimental time resolution was determined to be 50 fs by difference frequency generation of the $263 \mathrm{~nm}$ pump and the $395 \mathrm{~nm}$ probe pulse in a $100 \mu \mathrm{m}$ BBO crystal for the FEMTOPRO systems. To simulate the experimental conditions, a $1 \mathrm{~mm}$ quartz plate was placed in front of the crystal to mimic the quartz window of the sample cell. The time resolution of the UV-NOPA pumped by the CPA 2001 was estimated to be 50 fs from the ultrafast onset of the ground state bleaching of p-terphenyl that was compared with results obtained with the FEMTOPRO system.

All experiments were performed in the liquid phase with cyclohexane as solvent at room temperature. For the investigations of CHT a flow cell system was used to ensure that the sample volume was replenished between two consecutive laser shots. CHTd8, however, was only available in a small amount and a standing cuvette had to be used instead. Under the experimental conditions no difference could be found in the transients recorded in the flow cell and the standing cuvette in CHT and therefore, we expect the same for CHTd8. Nevertheless, the exposure time was kept as low as possible and the position of the CHTd8 sample was changed frequently by moving the cuvette vertically. Concentrations of CHT and CHTd8 of $0.02 \mathrm{~mol} / \mathrm{l}$ were prepared to ensure that the pump beam at $263 \mathrm{~nm}$ was completely (> $99 \%$ ) absorbed within a thickness of $<0.1 \mathrm{~mm}$ [5]. Such high concentrations prevent a noticeable group velocity mismatch between pump and probe pulses within the sample.

\section{Results}

Wavelength dependent pump-probe experiments for CHT with $\lambda_{\text {pump }}=263$ $\mathrm{nm}$ and $\lambda_{p r}$ between 373 and $298 \mathrm{~nm}$ are shown in Fig. 2. The fits are according to the scheme in Ref. [9]:

$$
\begin{gathered}
\mathrm{CHT} \stackrel{\tau_{0}}{\longrightarrow} \mathrm{CHT}^{\dagger} \\
\mathrm{CHT}^{\dagger} \stackrel{\tau_{I C}}{\longrightarrow} \mathrm{CHT}^{*} \\
\mathrm{CHT}^{*} \stackrel{\tau_{v i b}}{\longrightarrow} \mathrm{CHT}
\end{gathered}
$$

Here, $\tau_{0}$ denotes the time resolution of the experiment, $\tau_{I C}$ is the time constant for the internal conversion and $\tau_{v i b}$ describes vibrational cooling. Assuming exponential decays for the individual processes the values for $\tau_{I C}$ are found to be independent of the probe wavelength with values of $\tau_{I C}^{C H T}=(100 \pm 10)$ fs and $\tau_{I C}^{C H T d 8}=(150 \pm 20)$ fs in accordance with Ref. [9]. As expected, $\tau_{I C}^{C H T}<\tau_{I C}^{C H T d 8}$ due to the isotope effect [9]. The absolute time constants 


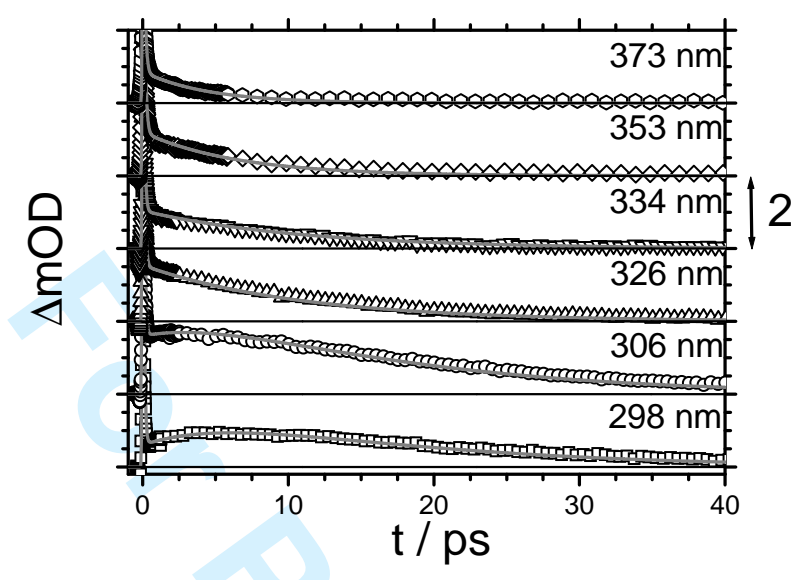

Figure 2: Pump-probe experiment of CHT with $\lambda_{\text {pump }}=263 \mathrm{~nm}$ and $\lambda_{p r}$ between 298 and $373 \mathrm{~nm}$ highlighting the regime of vibrational relaxation. The length of the arrow corresponds to $2 \Delta \mathrm{mOD}$.

are slightly shorter than reported previously due to polarization effects on a 100 fs timescale.

The wavelength dependent measurements show a slower decay with decreasing probe wavelengths which finally results in a local maximum at $\lambda_{p r}=298$ $\mathrm{nm}$ at a delay time of $\mathrm{t} \sim 5.3 \mathrm{ps}$ (see Fig. 3). In the following, we show how time constants for vibrational cooling can be extracted:

Under certain constraints it is expected that after photo-excitation and internal conversion a microcanonical ensemble of vibrationally excited molecules in the electronic ground state is obtained. This causes a residual absorption on the ps timescale whereas CHT at room temperature does not significantly absorb between 320 and $395 \mathrm{~nm}$. As has been demonstrated, the absorption spectra of both thermally heated and photo-excited molecules corresponding either to a canonical or a microcanonical energy distribution can be represented by the modified Sulzer-Wieland approach (see appendix). The spectra of canonical and microcanonical distributions are then very similar or even indistinguishable in the limit of identical average excitation energies. This can be easily rationalized since the population of the lowest vibrational levels of a microcanonical and a canonical distribution are identical for polyatomic molecules at sufficiently high excitation energies [11]. Moreover, the Sulzer-Wieland approach can be transferred to polyatomic molecules using a pseudodiatomic description. We have adapted this formalism to analyze the transient absorption of CHT. Transient spectra of CHT could be retrieved from Fig. 2 by identifying the maximum position at $298 \mathrm{~nm}$ and a delay time of $5.3 \mathrm{ps}$ as the maximum of a transient spectrum (see Fig. 5). In this way, a conversion to an $\epsilon(t)$ plot can be obtained where $\epsilon$ represents the molar decadic absorption coefficient of CHT. However, for CHTd8 such a pronounced point was not found presumably due to the 


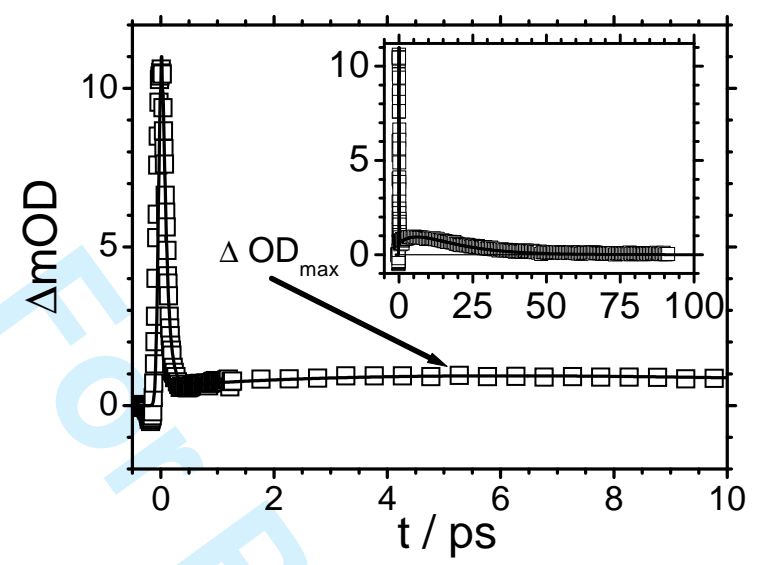

Figure 3: Pump-probe experiment of CHT with $\lambda_{p u m p}=263 \mathrm{~nm}$ and $\lambda_{p r}=298 \mathrm{~nm}$. The inset shows the response on a longer timescale.

blue-shift of the steady-state spectrum (see Fig. 4 for the pump-probe measurements); unfortunately, a transient response could not be obtained below 298 $\mathrm{nm}$ both for CHT and CHTd8 because the probe pulse was strongly absorbed. Consequently, transient spectra will be given for CHT only using the following equation:

$$
\epsilon(t)=\epsilon(\infty)+\frac{\Delta \mathrm{OD}(t)}{\Delta \mathrm{OD}(5.3 p s)}(\epsilon(5.3 p s)-\epsilon(\infty)) .
$$

Here, $\epsilon(\infty)$ corresponds to the absorption coefficient of the equilibrated absorption spectrum at room temperature $(3100 \mathrm{l} /(\mathrm{mol} \cdot \mathrm{cm}))[22]$ and $\epsilon(5.3 \mathrm{ps})$ is the absorption coefficient at $5.3 \mathrm{ps}$. At $298 \mathrm{~nm}$ we have $\epsilon(5.3 \mathrm{ps})=\epsilon_{\max }$ and $\Delta \mathrm{OD}(5.3 \mathrm{ps})=\Delta \mathrm{OD}_{\max }$, respectively, when neglecting the first maximum at about 50 fs that is due to the internal conversion. Using Eq. (4) all transients from Fig. 2 were recalculated and transient spectra at different delays were obtained (see Fig. 5).

The calculation of the transient spectra was performed according to a modified Sulzer-Wieland ansatz using Eqs. (8) and (9) (see appendix). The absorption spectrum of CHT has two maxima in the UV (see Fig. 1) which are considered via $\epsilon(\nu, T)=\epsilon_{1}(\nu, T)+\epsilon_{2}(\nu, T)$. For the elucidation of transient spectra only the maximum at $261 \mathrm{~nm}$ could be resolved in the absorption spectrum (Fig. 1); the second transition has its maximum below $200 \mathrm{~nm}$ which could not be resolved with our spectrometer. Therefore, the asymmetry inserted by Eq. (9) was only considered for the transition at $261 \mathrm{~nm}\left(38314 \mathrm{~cm}^{-1}\right)$ while for the second transition no asymmetry was taken into account. This is justified because the spectral contribution of the second transition in the experimental accessible region between 300 and $400 \mathrm{~nm}$ is expected to be small. The parameters in Eq. (8) for CHT have been adapted from Ref. [23]; to account for the 


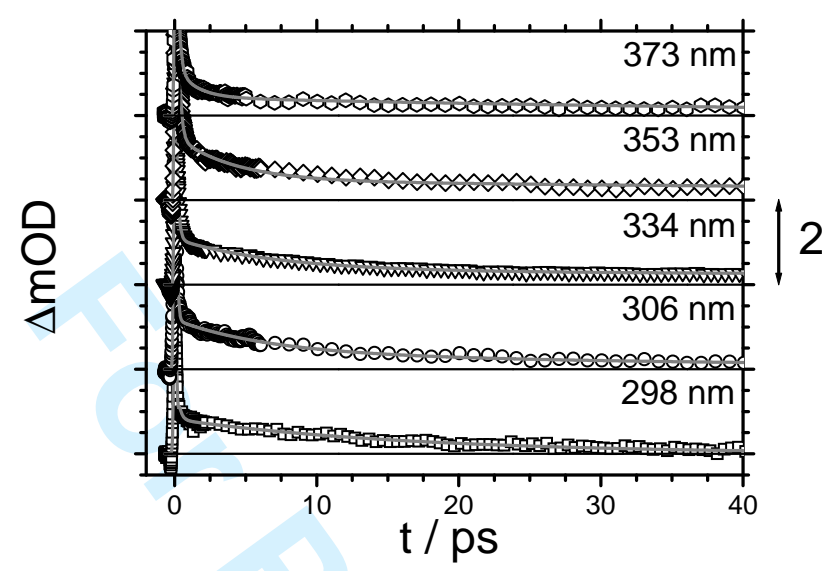

Figure 4: Pump-probe experiment of CHTd8 with $\lambda_{p u m p}=263 \mathrm{~nm}$ and $\lambda_{p r}$ between 298 and $373 \mathrm{~nm}$ highlighting the regime of vibrational relaxation. The length of the arrow corresponds to $2 \Delta \mathrm{mOD}$.

spectral red-shift in cyclohexane compared to gas phase data the parameter $\nu_{0}$ has been changed from 39300 (gas phase) to $38314 \mathrm{~cm}^{-1}$ (liquid phase: cyclohexane). The experimental data were evaluated according to Eq. (4) and are also shown for specific delay times. Fig. 5 clearly demonstrates that after 500 fs the temporal evolution of CHT can be understood in terms of vibrational relaxation that can be described by the modified Sulzer-Wieland formalism (see section 4 for details). At earlier times (hexagons in the inset of Fig. 5, representing a delay of $100 \mathrm{fs}$ ) no agreement between calculations and experimental results can be found. This indicates that another state rather than the "hot" ground state is observed and consequently must be assigned to spectral properties of the internal conversion. The absorption coefficient given at $100 \mathrm{fs}$ are only approximate values because of polarization effects.

According to our analysis, each delay time can be quantitatively simulated by a modified Sulzer-Wieland formalism that describes spectra at different temperatures. Therefore, one can assign each delay to a certain temperature. On the other hand, it is known (see, e.g., textbooks of statistical mechanics) that the temperature can be related to the vibrational energy $\mathrm{U}_{\text {vib }}(\mathrm{T})$ via

$$
U(T)=\sum_{i=1}^{n_{v}} \frac{R \Theta_{i}}{\exp \left\{\frac{\Theta_{i}}{T}\right\}-1}
$$

where $i$ denotes the different vibrational frequencies as given in Ref. [23] and $\Theta_{i}$ are the corresponding vibrational temperatures (see appendix). As a result, we obtain a relationship between delay time and $\mathrm{U}_{v i b}(\mathrm{~T})$ as displayed in Fig. 6. A simple monoexponential fit gives a decay time of $(8.5 \pm 1)$ ps which is assigned to the vibrational relaxation time of CHT in cyclohexane. This time constant is in reasonable agreement with investigations in other solvents like ethanol and 


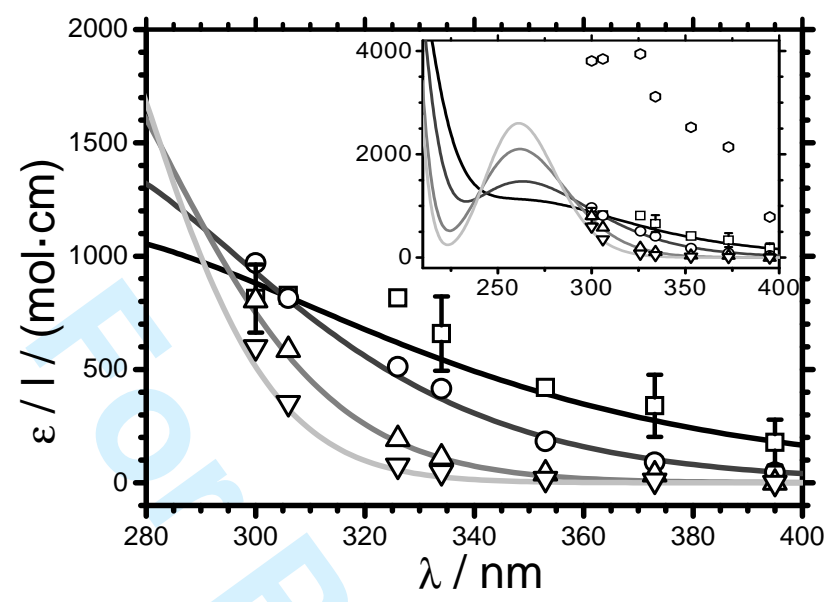

Figure 5: Transient spectra of CHT in cyclohexane, constructed with the method described in section 3. The symbols represent delay times of $\square: 500 \mathrm{fs}, \bigcirc: 5 \mathrm{ps}, \triangle: 20 \mathrm{ps}, \nabla: 90 \mathrm{ps}$. The curves are fits according to the modified Wieland-Sulzer ansatz at $\mathrm{T}=2200 \mathrm{~K}$ (black), $1200 \mathrm{~K}$ (dark gray), $500 \mathrm{~K}$ (gray) and $300 \mathrm{~K}$ (light gray). In the inset, the spectrum between 220 and $400 \mathrm{~nm}$ is shown. Added are the extinction coefficients at $\tau=100 \mathrm{fs}$ (hexagons).

heptane where relaxation times of $7.9 \mathrm{ps}$ and $9.5 \mathrm{ps}$ respectively were obtained by Benzler et. al. $[13,14]$ who used a slightly higher excitation energy $(41,000$ $\mathrm{cm}^{-1}$ compared to $38000 \mathrm{~cm}^{-1}$ in our studies).

\section{Discussion}

In most publications on pump-probe studies in liquid phase, the contribution of vibrational relaxation is fitted in terms of one exponential or the sum of several exponential decays. The decay constant for this process is thought to depend on the probe wavelength: the shorter the wavelength, the longer the time constant (for a more thorough explanation see, e.g., ref. [24]). The same effect can be observed for CHT: Fitting the data to a three-exponential decay function as described above, where the first accounts for the $\mathrm{H}$-shift and the last mostly describes a small offset (except for the measurements at 306 and $298 \mathrm{~nm}$ ) leads to results with small $\chi^{2}$-values. However, when considering the residuals of the transients of CHT after subtraction from the three-exponential fit (see Fig. 7) one observes a wavelength independent deviation from the exponential behavior indicating a general error when using this fitting function. These deviations can be explained by two factors:

(i) Instead of constructing transient spectra and comparing them to spectra obtained by the Sulzer-Wieland approach, it is possible to use a global fitting procedure to receive the time constant for vibrational cooling. The appropriate decay function is given by: 


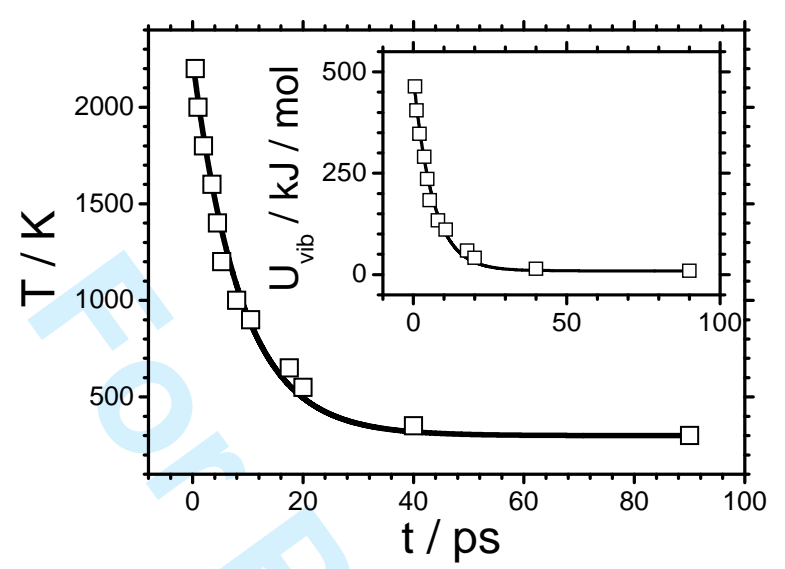

Figure 6: Time dependence of vibrational cooling of CHT in cyclohexane following excitation at $\lambda=263 \mathrm{~nm}$.

$$
\begin{aligned}
& f(t)=C_{1} \exp \left\{-t / \tau_{I C}\right\}+C_{2}[\epsilon(b, 300 \mathrm{~K}+ \\
& \left.\left.1900 \mathrm{~K} \exp \left\{-t / \tau_{v i b}\right\}\right)-\epsilon(b, 300 \mathrm{~K})\right]+C_{3},
\end{aligned}
$$

where $\epsilon$ is used as defined in Eq. (8) and $b=\left(\frac{\nu-\nu_{0}}{\Delta \nu_{0}}\right)^{2}$ (see appendix for a detailed explanation of the used parameters). The fitting parameters according to a Levenberg-Marquardt algorithm are $\Theta=420 \mathrm{~K}$ and $\tau_{v i b}=$ 10.3 ps which are in good argreement with $\Theta \approx 430 \mathrm{~K}$ as proposed by Hippler et al. [11] and used in the former analysis. The proposed decay according to Eq. (6) removes the wavelength independent structure of the residuals except the first extremum around $t=450 \mathrm{fs}$ (see (ii)). Although neither the time constant for vibrational relaxation nor the parameter $\Theta$ deviate markedly from the former analysis and is in accordance with former publications, the method should not be used without care: In most experiments, vibrational relaxation can only be analyzed by a decaying slope rather than by constructing a whole spectrum. Considering only the measurements for $\lambda \geq 326 \mathrm{~nm}$, the global fitting procedure delivers a time constant of $\tau_{v i b} \approx 23$ ps and $\Theta>1000 \mathrm{~K}$ since the region of a $\Delta$ OD-rise is obviously wrongly predicted, eventually due to the signal to noise ratio or because the Sulzer-Wieland ansatz is not good enough. Hence, these parameters do not allow to obtain the appropriate behavior for shorter wavelengths. As a consequence, no comparable quantitative analysis for the CHTd8-system can be given.

(ii) As noted above, the first maximum of the residuals around 450 fs does not vanish when using Eq. (6) instead of an exponential decay for wavelengths 
of $\lambda \geq 326 \mathrm{~nm}$. For 306 and $298 \mathrm{~nm}$, the residuals exibit negative values. This behavior can be explained as follows: According to Fig. 5, a higher $\Delta$ OD-value corresponds to a higher temperature for $\lambda \geq 326 \mathrm{~nm}$ while for 306 and $298 \mathrm{~nm}$ a higher temperature is indicated by a lower $\Delta \mathrm{OD}$-value. In consequence, the residuals indicate a higher initial temperature than the one obtained by the exponential fit. There are at least two possible reasons for this behavior:

(iia) During the first few hundreds femtoseconds the molecules are supposed to rotate freely as can be studied by, e.g., transient anisotropy (see Ref. [25] and references cited therein). Moreover, the average effective collision time of CHT in various solvents was found to be 2$2.5 \mathrm{ps}^{-1}$ by Benzler et. al. [14], which coincides with the delay time of the extremum. Therefore, one may conclude that the molecule remains its initial temperature until its first collision. The higher temperature is therefore due to non-statistical collision processes at short time delays.

(iib) The initial energy distribution of the molecules is not canonical. This might affect the probe process since no molecule has initially an internal energy that is below the threshold of being probed. Therefore, the experiment might overestimate the initial temperature.

As mentioned, collision induced vibrational cooling of CHT has already been investigated by Benzler et al. [13, 14] (see sections 1 and 3). The authors used pump-probe experiments with a time resolution of $500 \mathrm{fs}, \lambda_{\text {pump }}=248 \mathrm{~nm}$ 
and $\lambda_{\text {probe }}=225$ and $313 \mathrm{~nm}$. In order to extract the time dependence of the cooling, hot spectra from shock tube data [12] have been interpolated to higher temperatures. While this method seems to be an efficient way to extract the time dependence of collisional energy transfer, the presented results offer additional information:

(a) Transient spectra have been extracted and adopted to $\epsilon(t)$-values (mostly, the relative change in optical density is measured without absolute values).

(b) The time dependent spectra could be associated with a (vibrational) temperature by the modified Sulzer-Wieland ansatz. While this model includes several assumptions, it is sufficient to describe spectra within the uncertainties of the measurement. Other models based on spectral moments have been given [26]-[30]; however, the modified Sulzer-Wieland ansatz has the advantage of an analytic expression and a small set of adjustable parameters.

(c) For this analysis it is necessary to identify a (local) maximum in the $\Delta \mathrm{OD}(t)$-measurements at a certain probe wavelength - in the case of $\mathrm{CHT}$ at $\lambda_{\text {probe }}=298 \mathrm{~nm}$ that can be assigned to the cooling process. It is expected that such extrema should occur on a ps timescale in many systems and hence, should be far away from cross correlation contributions around time zero supposed the time resolution is high enough. In any case, this local extremum is fundamental for the construction of the $\epsilon(t)$ spectra. One of the main results of this analysis is that local extrema in ultrafast absorption spectroscopy do not necessarily have to be assigned to additional time constants and physical processes. As shown, vibrational cooling in the electronic ground state has also to be considered.

(d) A sufficient time resolution allows for the observation of non-statistical effects in an otherwise exponential temperature decay.

While the presented method gives a lot of information concerning vibrational cooling, there are as well several problems which can be seen in the case of CHTd8: Here, there neither exist parameters for the modified Sulzer-Wieland model (i.e., no temperature dependent spectra) nor a maximum in the $\Delta \mathrm{OD}(\mathrm{t})$ curve to extract transient spectra. Therefore, little information on a quantitative level for the vibrational cooling of this molecule can be obtained.

Finally, it is worth to briefly compare the presented experiments with the investigation of vibrational cooling in IR-pump Raman-probe studies (see Refs. [31][33] and references cited therein). In these kind of experiments, an IR-photon is used to excite a high frequency mode (normaly a CH-strech-mode). Afterward, a narrow bandwidth VIS-pulse induces Raman scattering which allows to record the time dependent population of various vibrational modes. This powerful technique offers the possibility to study vibrational cooling as well as internal vibrational redistribution on a ps-timescale. However, the results presented so far only have a time resolution of $\sim 1 \mathrm{ps}$ and it is uncertain whether the dynamics 
following excitation of a single mode are able to explain the ground state dynamics following UV-irradiation and ultrafast internal conversions. The often used three stage model to describe Raman experiments [32]-[34] is a microcanonical picture, i.e., it keeps track of every vibrational mode. In order to describe highly excited molecules, especially those with many vibrational degrees of freedom, the introduction of a canonical picture (and hence a vibrational temperature as the only defining parameter of the system) seems adequate. This offers a simple model of the underlying dynamics.

\section{Conclusion}

In this work, we have performed pump-probe investigations of CHT and CHTd8 in cyclohexane with a time resolution of $50 \mathrm{fs}$ at probe wavelengths between 300 and $400 \mathrm{~nm}$ after excitation to the $S_{2}$-state with $\lambda_{\text {pump }}=263 \mathrm{~nm}$ in order to study vibrational cooling on an ultrafast timescale. After internal conversion within the first hundreds of $\mathrm{fs}$, the molecule resides in the vibrationally excited electronic ground state. Using results of earlier works on microcanonical and canonical energy distributions, we could extract and simulate transient spectra of CHT by applying a modified Sulzer-Wieland equation. As a result, each delay time ( $\geq 500 \mathrm{fs}$ ) in the transient absorption profiles that monitors a microcanonical distribution can be interpreted in terms of a canonical distribution at a specific temperature and hence a vibrational energy. From this we obtained a time constant for the vibrational relaxation $\tau_{v i b}$ for $\mathrm{CHT}$ of $8.5 \mathrm{ps}$ in cyclohexane as solvent.

Acknowledgments This work has been financially supported by the Deutsche Forschungsgemeinschaft (DFG) and the University of Karlsruhe (TH).

\section{A. Sulzer-Wieland ansatz}

\section{A.1. Basic equations}

In the classical Sulzer-Wieland approach the upper state potential is assumed to be repulsive and without curvature. Moreover, the oscillator strength of a transition does not depend on the temperature such that the extinction coefficient can be expressed by $[15,35]$

$$
\frac{\epsilon(\nu, T)}{\nu} \stackrel{\propto}{\sim} \epsilon(\nu, T)=\epsilon_{0}^{\max } \sum_{n=0}^{\infty} N_{n}(T)\left|\Psi_{n}(\nu)\right|^{2}
$$

where $\epsilon_{0}^{\max }$ is the extinction coefficient at zero temperature, $\Psi_{n}(\nu)$ is the $n$-th vibrational wave function of the electronic ground state and $N_{n}(T)$ is its occupation number. In the Sulzer-Wieland model the ground state is described by a harmonic oscillator whose wave function is given by $\Psi_{n}^{\text {harm }}(\xi)=G_{n} e^{-\xi / 2} H_{n}(\xi)$, where $\xi=\left(\nu-\nu_{0}\right) / \Delta \nu_{0}, \nu_{0}$ is the mean transition energy $\left[\mathrm{s}^{-1}\right], G_{n}$ the normalization constant and $H_{n}$ the $n$-th Hermite polynomial. The factor $\Delta \nu_{0}$ is 
given by the slope of the upper state potential. The occupation number for a harmonic potential is given by $N_{n}^{\text {harm }}=(1-z) z^{n}$, where $z=\exp \{-\Theta / T\}$ with $\Theta=\hbar \omega_{0} / k_{B}$. The factor $\nu$ on the left hand side of Eq. (7) is neglected. Then, the temperature dependence can be calculated to give

$$
\begin{aligned}
& \epsilon(\nu, T)=\epsilon_{0}^{\max }\left[\tanh \left(\frac{\Theta_{0}}{2 T}\right)\right]^{\frac{1}{2}} \times \\
& \exp \left\{-\tanh \left(\frac{\Theta_{0}}{2 T}\right)\left(\frac{\nu-\nu_{0}}{\Delta \nu_{0}}\right)^{2}\right\}
\end{aligned}
$$

Two extensions are given to account for a red-shift of the absorption spectra with increasing temperature and for an asymmetry of the recorded spectra $[12,11]$ :

1. In the first extension, Eq. 8 is multiplied by $\nu / \nu_{0}$ and the frequency $\nu_{0}$ is modified through the average vibrational energy of the $\omega_{0}$-vibration:

$$
\nu_{0, e f f}=\nu_{0}-\frac{\omega_{0}}{\exp \{\Theta / T\}-1}
$$

2. If more than one spectral change inducing mode is present, the SulzerWieland equation (8) can be modified by

$$
\epsilon(\nu, T)=\epsilon^{S W}(\nu, T) \frac{\nu}{\nu_{0}}\left(1+A \xi+B \xi^{3}\right),
$$

where $A$ and $B$ are parameters depending on $z, \omega_{0}$ and $\Delta \nu_{0}$ only (see Ref. [11]).

\section{A.2. Multidimensional Sulzer-Wieland ansatz}

The classical Sulzer-Wieland ansatz was originally developed for diatomic molecules exhibiting a single vibration that is treated in the approximation of a harmonic oscillator. However, a polyatomic molecule exhibits a density of vibrational states that is much different from a diatomic. The differences between the classical and a multidimensional model are shortly discussed in the following:

For a polyatomic molecule, the Sulzer-Wieland equation can be written as

$$
\epsilon_{\text {poly }}(\nu, T)=\epsilon_{0}^{\max } \sum_{i=1}^{n_{v}} \sum_{n=0}^{\infty} N_{n, i}(T)\left|\Psi_{n, i}(\nu)\right|^{2}
$$

where $i$ runs over all vibrations of the molecule and the occupation number is given by $N_{n, i}(T)=\exp \left\{-n \Theta_{i} / T\right\} /\left(\sum_{i, n} \exp \left\{-n \Theta_{i} / T\right\}\right)$. Here, $\Theta_{i}$ are the vibrational temperatures for the $i$-th vibrational mode. This model implies that all wavefunctions can be reflected at the same slope of the upper potential energy surface (idealized as a straight line). Fig. 8 shows plots at different temperatures where the vibrational frequencies of CHT were used to calculate $\epsilon_{\text {poly }}(\nu, T)$. These plots are compared with the results obtained via the one 


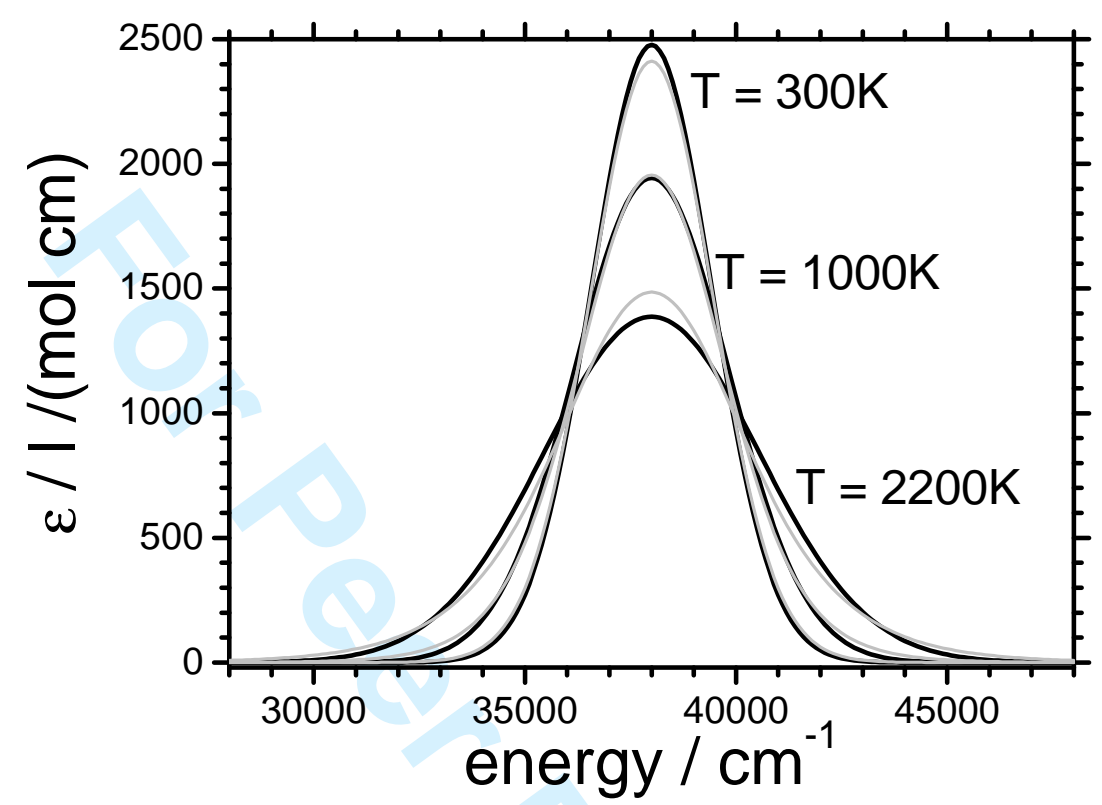

Figure 8: Comparison of the temperature dependency of the absorption spectra according to a one dimensional Sulzer-Wieland model (black curves) and the multidimensional treatment (gray curves) for different temperatures. The chosen parameters were $\nu_{0}=38,000 \mathrm{~cm}^{-1}$ and $\Delta \nu=2,000 \mathrm{~cm}^{-1}$. The $\Theta_{i}$ are the values for CHT (see [23]).

dimensional Sulzer-Wieland approach. As can be seen, the one dimensional formula is able to represent the multidimensional model for an adequate choice of $\Theta_{0}$ at least up to a temperature of $2200 \mathrm{~K}$. The shapes of the spectra are slightly different, nevertheless, it seems justified to work with a one-dimensional representation. 


\section{References}

[1] P. M. Borell, H.-G. Löhmannsröben, K. Luther, Chem. Phys. Lett. 136 (1987) 371.

[2] P. J. Reid, A. P. Shreve, R. A. Mathies, J. Phys. Chem. 97 (1993) 12691.

[3] P. J. Reid, M. K. Lawless, S. D. Wickham, R. A. Mathies, J. Phys. Chem. 98 (1994) 5597.

[4] M. K. Lawless, S. D. Wickham, R. A. Mathies, Acc. Chem. Res. 28 (1995) 493.

[5] A. Hertwig, H. Hippler, H. Schmid, A. N. Unterreiner, Phys. Chem. Chem. Phys. 1 (1999) 5129.

[6] S. A. Trushin, S. Diemer, W. Fuß, K. L. Kompa,W. E. Schmid, Phys. Chem. Chem. Phys. 1 (1999) 1431.

[7] H.-M. Steuhl, C. Bornemann, M. Klessinger, J. Chem. Eur. 5 (1999) 2404.

[8] C. Y. Ruan, V. A. Lobastov, R. Srinivasan, B. M. Goodson, H. Ihee, A. H. Zewail, PNAS 98 (2001) 7117.

[9] H. Hippler, M. Olzmann, O. Schalk, A. N. Unterreiner, Z. Phys. Chem. 219 (2005) 389.

[10] O. Schalk, A.-N. Unterreiner, J. Phys. Chem. A 111 (2007) 3231.

[11] H. Hippler, K. Luther, J. Troe, H. J. Wendelken, J. Phys. Chem. 79 (1983) 239.

[12] D. C. Astholz, L. Brouwer, J. Troe, Ber. Bunsenges. Phys. Chem. 85 (1981) 559.

[13] J. Benzler, S. Linkersdörfer, K. Luther, Ber. Bunsenges. Phys. Chem. 100 (1996) 1252.

[14] J. Benzler, S. Linkersdörfer, K. Luther, J. Phys. Chem. 106 (1997) 4992.

[15] P. Sulzer, K. Wieland, Helv. Phys. Acta 85 (1952) 559.

[16] C. Engdahl, G. Jonsäll, P. Ahlberg, J. Am. Chem. Soc. 105 (1083) 891.

[17] M. Lenzner, C. Spielmann, E. Winter, F. Krausz, A. J. Schmidt, Opt. Lett. 20 (1995) 1397.

[18] S. Sartania, Z. Cheng, M. Lenzner, G. Tempea, C. Spielmann, F. Krausz, K. Ferencz, Opt. Lett. 22 (1997) 1562.

[19] A.-N. Unterreiner, PhD thesis, Universität Karlsruhe (TH), 1998, ISBN: 3-89653-368-1. 
[20] T. Wilhelm, J. Piel, E. Riedle, Opt. Lett. 22 (1997) 1494.

[21] J. Piel, M. Beutter, E. Riedle, Opt. Lett. 25 (2000) 180.

[22] R. P. Frueholz, R. Rianda, A. Kuppermann, Chem. Phys. Lett. 55 (1978) 88.

[23] D. C. Astholz, J. Troe, W. Wieters, J. Chem. Phys. 70 (1979) 5107.

[24] O. Schalk, H. Brands, T. S. Balaban, A.-N. Unterreiner, J. Phys. Chem. A 112 (2008) 1719.

[25] O. Schalk, A.-N. Unterreiner, J. Chem. Phys. (submitted).

[26] E. A. Gislason, J. Chem. Phys. 58 (1973) 3702.

[27] S. M. Adler-Golden, Chem. Phys. 64 (1982) 421.

[28] J. A. Joens, Chem. Phys. Lett. 138 (1987) 512.

[29] J. A. Joens, J. Phys. Chem. 97 (1993) 2527.

[30] R. D. Coalson, M. Karplus, J. Chem. Phys. 81 (1984) 2891.

[31] S. Shigeto, D. D. Dlott, Chem. Phys. Lett. 447 (2007) 134.

[32] S. Shigeto, Y. Pang, Y. Fang, D. D. Dlott, J. Phys. Chem. B 112 (2008) 232.

[33] N.-H. Seong, Y. Fang, D. D. Dlott, J. Phys. Chem. A 113 (2009) 1445.

[34] A. Nitzan, J. Jortner, Mol. Phys. 25 (1973) 713.

[35] G. Herzberg, Molecular Spectra of Molecular Structure, Vol. 3, Van Nostrand Reinhold, New York (1966). 


\section{List of Figures}

1 UV-Vis spectra of CHT and CHTd8 in cyclohexane, adopted from Ref. [9]. . . . . . . . . . . . . . . . . . . . . .

2 Pump-probe experiment of CHT with $\lambda_{\text {pump }}=263 \mathrm{~nm}$ and $\lambda_{p r}$ between 298 and $373 \mathrm{~nm}$ highlighting the regime of vibrational relaxation. The length of the arrow corresponds to $2 \Delta$ mOD. . . 5

3 Pump-probe experiment of CHT with $\lambda_{\text {pump }}=263 \mathrm{~nm}$ and $\lambda_{p r}=$ $298 \mathrm{~nm}$. The inset shows the response on a longer timescale. . . 6

4 Pump-probe experiment of CHTd8 with $\lambda_{\text {pump }}=263 \mathrm{~nm}$ and $\lambda_{p r}$ between 298 and $373 \mathrm{~nm}$ highlighting the regime of vibrational relaxation. The length of the arrow corresponds to $2 \Delta \mathrm{mOD}$. . .

5 Transient spectra of CHT in cyclohexane, constructed with the method described in section 3 . The symbols represent delay times of $\square: 500 \mathrm{fs}, \bigcirc: 5 \mathrm{ps}, \triangle: 20 \mathrm{ps}, \nabla: 90 \mathrm{ps}$. The curves are fits according to the modified Wieland-Sulzer ansatz at $\mathrm{T}=2200 \mathrm{~K}$ (black), $1200 \mathrm{~K}$ (dark gray), $500 \mathrm{~K}$ (gray) and $300 \mathrm{~K}$ (light gray). In the inset, the spectrum between 220 and $400 \mathrm{~nm}$ is shown. Added are the extinction coefficients at $\tau=100 \mathrm{fs}$ (hexagons).

6 Time dependence of vibrational cooling of CHT in cyclohexane following excitation at $\lambda=263 \mathrm{~nm} . \ldots \ldots \ldots$

7 Residuals of the transients of CHT after subtraction from a threeexponential fit for a pump wavelength of $263 \mathrm{~nm}$ and various probe wavelength $(298,326,334,353$ and $373 \mathrm{~nm})$. In the inset, the average over the residuals of the four longest wavelengths are shown. See text for discussion.

8 Comparison of the temperature dependency of the absorption spectra according to a one dimensional Sulzer-Wieland model (black curves) and the multidimensional treatment (gray curves) for different temperatures. The chosen parameters were $\nu_{0}=$ $38,000 \mathrm{~cm}^{-1}$ and $\Delta \nu=2,000 \mathrm{~cm}^{-1}$. The $\Theta_{i}$ are the values for CHT (see [23]). 


\title{
Vibrational cooling in the liquid phase studied by ultrafast investigations of cycloheptatriene
}

\author{
Oliver Schalk ${ }^{\mathrm{a}, \mathrm{b}}$, Ji-Ping Yang ${ }^{\mathrm{a}, \mathrm{c}}$, Andreas Hertwig ${ }^{\mathrm{a}, \mathrm{d}}$, Horst Hippler ${ }^{\mathrm{a}}$, \\ Andreas N. Unterreiner,a \\ ${ }^{a}$ Institut für Physikalische Chemie, Kaiserstr. 12, 76131 Karlsruhe, \\ Universität Karlsruhe (TH), Germany \\ ${ }^{b}$ present address: Steacie Institute for Molecular Sciences, National Research Council \\ Canada, Sussex Drive 100, Ottawa, Ontario, K1A 0R6, Canada \\ ${ }^{c}$ present address: Department of Applied Physics and Institute of Laser Technology, \\ Hefei University of Technology, Hefei, Anhui, Peop. Rep. China \\ ${ }^{d}$ present address: BAM, Div. VI.4 - Surface Technology, Unter den Eichen 87, 12205 \\ Berlin, Germany
}

\begin{abstract}
Solvent-mediated vibrational relaxation in UV pump-probe experiments has been studied using cycloheptatriene (CHT) and its perdeuterated counterpart $(\mathrm{CHTd} 8)$ as model systems. Transient spectra containing absolute $\epsilon(t)$-values are obtained by a modified Sulzer-Wieland ansatz and a global fit function for vibrational relaxation is proposed.
\end{abstract}

\section{Introduction}

Ultrafast relaxation of vibrationally excited molecules in the electronic ground state can be observed in many ultrafast pump-probe studies if a molecule is excited to a higher lying electronic state and subsequently returning to the ground state by one (or more) internal conversions to highly excited vibrationally states. Often, the energy dissipation to the solvent is treated only qualitatively lacking a detailed knowledge of the time-dependent evolution of the relevant absorption coefficients. In addition, photoexcitation may lead to a non-thermal energy distribution on an ultrashort timescale. For a more adequate description, additional information is then necessary. Here, we demonstrate that 1,3,5cycloheptatriene (CHT) may be regarded as a model system towards a more quantitative assignment of transient spectra including time-dependent absorption coefficients.

During the last decade, CHT and related compounds have been studied extensively by various spectroscopic techniques from nanosecond to femtosecond (fs) timescale [1]-[10]. From these studies, the following picture emerges: After

\footnotetext{
*Corresponding author

Email address: andreas.unterreiner@kit.edu (Andreas N. Unterreiner)
} 
excitation around $260 \mathrm{~nm}$ to the $S_{2}$-state, the molecule passes two conical intersections $\left(S_{1}-S_{2}\right.$ and $\left.S_{0}-S_{1}\right)$ and reaches the ground state in $\tau_{I C}=(110 \pm 15)$ fs $[5,6,9]$ with a vibrational temperature of $2200 \mathrm{~K}[8]$. Despite a typically quite narrow energy distribution that cannot be described canonically, it has been shown that the spectra of microcanonical and canonical distributions of the same temperature exhibit the same spectrum in second order perturbation theory [11]. Although there is some discrepancy in the literature concerning the timescale for the second intersection, a $[1,7]$-sigmatropic hydrogen migration is supposed to take place in the fs-regime as recently suggested by additional experiments on the perchlorinated counterpart of CHT [10]. Due to these fast internal conversions, CHT is ideally suited to study vibrational relaxation since no energy is dissipated to the surrounding solvent until reaching the electronic ground state. Necessary parameters for spectra reconstruction can be obtained from vibrational cooling studies after shock waves in the gaseous phase between 300 and $1200 \mathrm{~K}[12,11]$ and by pump-probe spectroscopy using 500 fs-pulses in compressed gases, supercritical fluids and liquids $[13,14]$. The spectra obtained from shock wave experiments have been explained by means of a modified Sulzer-Wieland ansatz [15] while pump-probe experiments were used to extract the energy loss due to collisions in media of different densities.

In this paper, we show that the modified Sulzer-Wieland approach can be transfered to femtosecond time resolved measurements to construct transient spectra with quantitatively determined absorption coefficients $\epsilon(t)$ after excitation of CHT and present a method to fit the vibrational cooling observed in the transients of pump-probe experiments.

\section{Experimental methods}

\subsection{Sample preparation}

CHT was used as obtained from Fluka with a nominal purity of $>97 \%$ and CHTd8 has been synthesized by Dr. Eckert GmbH (Germany) according to the procedure described by Engdahl et al. [16]. The purities (about $90 \%$ for CHTd8) [9] have been checked by ${ }^{1} \mathrm{H}-\mathrm{NMR}$ and ${ }^{13} \mathrm{C}-\mathrm{NMR}$ in $\mathrm{CDCl}_{3}$ (Bruker, $250 \mathrm{MHz}$ ), UV-vis spectroscopy (Varian, Cary 5e) and GC-MS for CHTd8. The UV-vis spectra for CHT and CHTd8 are shown in Fig. 1. The spectrum of CHT is smooth and featureless as in essence holds for CHTd8, too. In addition, a small blue-shift of the absorption maximum of $3 \mathrm{~nm}$ compared to CHT is observed. A more detailed analysis showed that impurities in CHTd8 are mostly toluene and partially deuterated CHT (CHT-d6). Toluene gave a pump probe signal at $395 \mathrm{~nm}$ that was more than ten times smaller than for CHT and was therefore neglected. Also, no evidence of a contribution of partially deuterated CHT could be found.

\subsection{Experimental setup}

Two commercial laser systems with $1 \mathrm{kHz}$ repetition rate were used in this study: (i) a multi-pass amplifier system (FEMTOPRO, Femtolasers) with 30 fs 


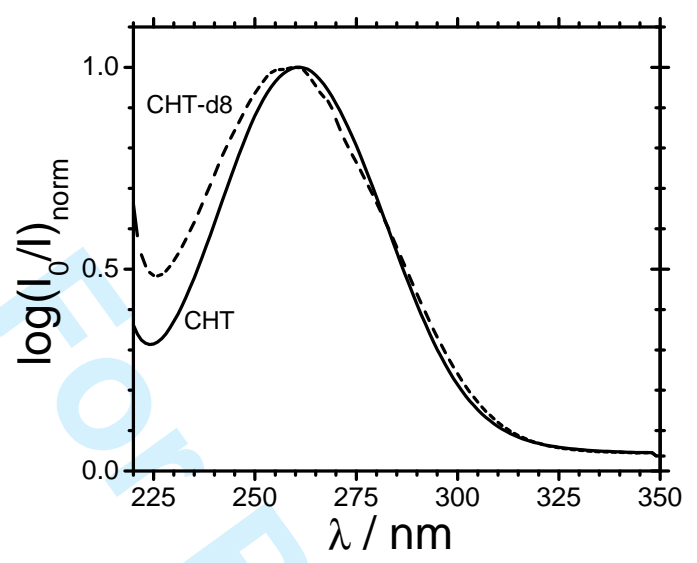

Figure 1: UV-Vis spectra of CHT and CHTd8 in cyclohexane, adopted from Ref. [9].

pulses at $790 \mathrm{~nm}[17,18]$ and (ii) a regenerative amplifier (CPA 2001, ClarkMXR) with $150 \mathrm{fs}$ pulses at $775 \mathrm{~nm}$.

The output of the multi-pass system was modified for pump probe spectroscopy by reducing the laser spot size by a factor of 3 to a diameter of 6 $\mathrm{mm}$ with two telescopes. Due to the high intensities the beam had to be split using a broad-band beam splitter and half of the output energy was used for further frequency conversion. The second harmonic was generated in a $100 \mu \mathrm{m}$ $\mathrm{BBO}$ crystal (type I) and separated by a dichroic mirror from the fundamental. By collinearly recombining the fundamental and the second harmonic light the third harmonic was generated in another $100 \mu \mathrm{m}$ BBO crystal (type I) and typically less than $2 \mu \mathrm{J}$ were used unfocused as pump pulse at $263 \mathrm{~nm}$. The 395 $\mathrm{nm}$ beam served as probe pulse after being attenuated to energies below $50 \mathrm{~nJ}$ by quartz plates. For a more detailed experimental setup see Ref. [19].

The regenerative amplifier served as a pump source for two independently tunable NOPA (noncollinear optical parametric amplifier) systems [20, 21]. The wavelength of one NOPA was set to $526 \mathrm{~nm}$ and frequency doubled to $263 \mathrm{~nm}$ with a typical energy of $0.5 \mu \mathrm{J}$ and served as pump pulse while the second NOPA was tuned between 298 and $373 \mathrm{~nm}$ at moderate energies below $10 \mathrm{~nJ}$. Pump and probe beams were directed separately onto two curved mirrors and focused into the sample. The polarization of the pulses were set parallel to avoid any additional dispersive elements in the beam path. Since it was shown that the anisotropy of these systems are nearly zero at delay times relevant for the the study of vibrational cooling ( $t \geq 500 \mathrm{fs}$ ) [9], no polarization effects need to be considered in the analysis.

Care was taken to ensure that the diameter of the pump pulse was always larger than the probe beam diameter. In both set-ups pump and probe beams intersected an angle of $4^{\circ}$ at the sample position. The pump beam was chopped 
at $500 \mathrm{~Hz}$ and temporally changed relative to the probe beam using a computer controlled translation stage (Melles Griot). Intensities of the probe pulse before and behind the cell were measured by photodiodes and the relative change of the optical density with and without the pump pulse, $\triangle \mathrm{OD}$, was recorded. To improve the signal-to-noise ratio time profiles were recorded by averaging 50 laser shots at each delay time for a single scan and 20 subsequent scans were averaged. The experimental time resolution was determined to be 50 fs by difference frequency generation of the $263 \mathrm{~nm}$ pump and the $395 \mathrm{~nm}$ probe pulse in a $100 \mu \mathrm{m}$ BBO crystal for the FEMTOPRO systems. To simulate the experimental conditions, a $1 \mathrm{~mm}$ quartz plate was placed in front of the crystal to mimic the quartz window of the sample cell. The time resolution of the UV-NOPA pumped by the CPA 2001 was estimated to be 50 fs from the ultrafast onset of the ground state bleaching of p-terphenyl that was compared with results obtained with the FEMTOPRO system.

All experiments were performed in the liquid phase with cyclohexane as solvent at room temperature. For the investigations of CHT a flow cell system was used to ensure that the sample volume was replenished between two consecutive laser shots. CHTd8, however, was only available in a small amount and a standing cuvette had to be used instead. Under the experimental conditions no difference could be found in the transients recorded in the flow cell and the standing cuvette in CHT and therefore, we expect the same for CHTd8. Nevertheless, the exposure time was kept as low as possible and the position of the CHTd8 sample was changed frequently by moving the cuvette vertically. Concentrations of CHT and CHTd 8 of $0.02 \mathrm{~mol} / \mathrm{l}$ were prepared to ensure that the pump beam at $263 \mathrm{~nm}$ was completely (> $99 \%$ ) absorbed within a thickness of $<0.1 \mathrm{~mm}[5]$. Such high concentrations prevent a noticeable group velocity mismatch between pump and probe pulses within the sample.

\section{Results}

Wavelength dependent pump-probe experiments for CHT with $\lambda_{\text {pump }}=263$ $\mathrm{nm}$ and $\lambda_{p r}$ between 373 and $298 \mathrm{~nm}$ are shown in Fig. 2. The fits are according to the scheme in Ref. [9]:

$$
\begin{gathered}
\mathrm{CHT} \stackrel{\tau_{0}}{\longrightarrow} \mathrm{CHT}^{\dagger} \\
\mathrm{CHT}^{\dagger} \stackrel{\tau_{I C}}{\longrightarrow} \mathrm{CHT}^{*} \\
\mathrm{CHT}^{*} \stackrel{\tau_{v i b}}{\longrightarrow} \text { CHT. }
\end{gathered}
$$

Here, $\tau_{0}$ denotes the time resolution of the experiment, $\tau_{I C}$ is the time constant for the internal conversion and $\tau_{v i b}$ describes vibrational cooling. Assuming exponential decays for the individual processes the values for $\tau_{I C}$ are found to be independent of the probe wavelength with values of $\tau_{I C}^{C H T}=(100 \pm 10)$ fs and $\tau_{I C}^{C H T d 8}=(150 \pm 20)$ fs in accordance with Ref. [9]. As expected, $\tau_{I C}^{C H T}<\tau_{I C}^{C H T d 8}$ due to the isotope effect [9]. The absolute time constants 


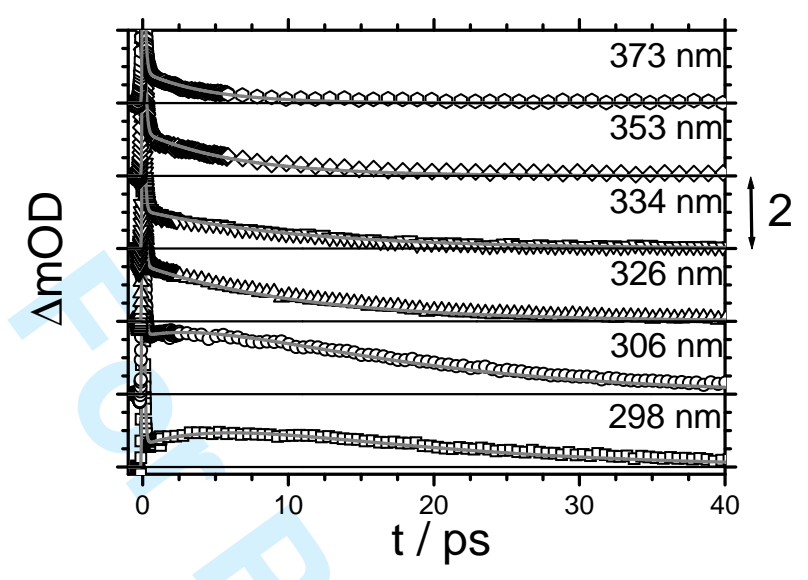

Figure 2: Pump-probe experiment of CHT with $\lambda_{p u m p}=263 \mathrm{~nm}$ and $\lambda_{p r}$ between 298 and $373 \mathrm{~nm}$ highlighting the regime of vibrational relaxation. The length of the arrow corresponds to $2 \Delta \mathrm{mOD}$.

are slightly shorter than reported previously due to polarization effects on a 100 fs timescale.

The wavelength dependent measurements show a slower decay with decreasing probe wavelengths which finally results in a local maximum at $\lambda_{p r}=298$ $\mathrm{nm}$ at a delay time of $\mathrm{t} \sim 5.3 \mathrm{ps}$ (see Fig. 3). In the following, we show how time constants for vibrational cooling can be extracted:

Under certain constraints it is expected that after photo-excitation and internal conversion a microcanonical ensemble of vibrationally excited molecules in the electronic ground state is obtained. This causes a residual absorption on the ps timescale whereas CHT at room temperature does not significantly absorb between 320 and $395 \mathrm{~nm}$. As has been demonstrated, the absorption spectra of both thermally heated and photo-excited molecules corresponding either to a canonical or a microcanonical energy distribution can be represented by the modified Sulzer-Wieland approach (see appendix). The spectra of canonical and microcanonical distributions are then very similar or even indistinguishable in the limit of identical average excitation energies. This can be easily rationalized since the population of the lowest vibrational levels of a microcanonical and a canonical distribution are identical for polyatomic molecules at sufficiently high excitation energies [11]. Moreover, the Sulzer-Wieland approach can be transferred to polyatomic molecules using a pseudodiatomic description. We have adapted this formalism to analyze the transient absorption of CHT. Transient spectra of CHT could be retrieved from Fig. 2 by identifying the maximum position at $298 \mathrm{~nm}$ and a delay time of $5.3 \mathrm{ps}$ as the maximum of a transient spectrum (see Fig. 5). In this way, a conversion to an $\epsilon(t)$ plot can be obtained where $\epsilon$ represents the molar decadic absorption coefficient of CHT. However, for CHTd8 such a pronounced point was not found presumably due to the 


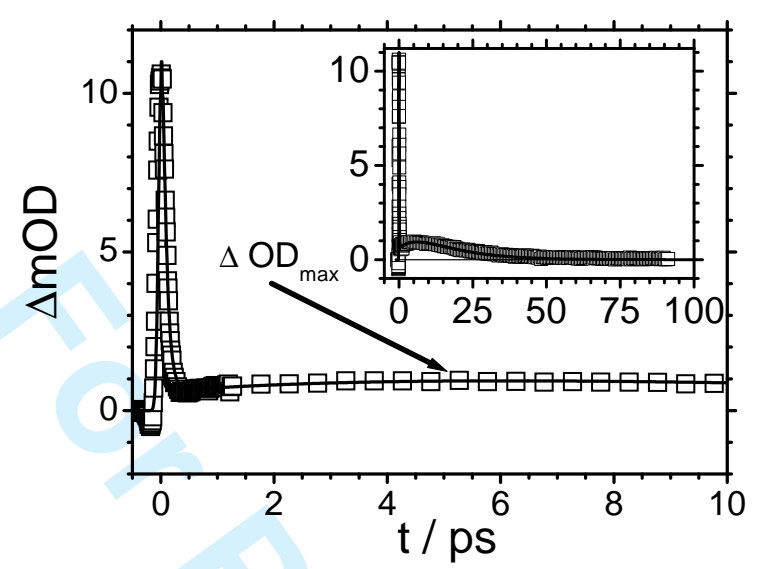

Figure 3: Pump-probe experiment of CHT with $\lambda_{p u m p}=263 \mathrm{~nm}$ and $\lambda_{p r}=298 \mathrm{~nm}$. The inset shows the response on a longer timescale.

blue-shift of the steady-state spectrum (see Fig. 4 for the pump-probe measurements); unfortunately, a transient response could not be obtained below 298 $\mathrm{nm}$ both for CHT and CHTd8 because the probe pulse was strongly absorbed. Consequently, transient spectra will be given for CHT only using the following equation:

$$
\epsilon(t)=\epsilon(\infty)+\frac{\Delta \mathrm{OD}(t)}{\Delta \mathrm{OD}(5.3 p s)}(\epsilon(5.3 p s)-\epsilon(\infty)) .
$$

Here, $\epsilon(\infty)$ corresponds to the absorption coefficient of the equilibrated absorption spectrum at room temperature $(3100 \mathrm{l} /(\mathrm{mol} \cdot \mathrm{cm}))[22]$ and $\epsilon(5.3 \mathrm{ps})$ is the absorption coefficient at $5.3 \mathrm{ps}$. At $298 \mathrm{~nm}$ we have $\epsilon(5.3 \mathrm{ps})=\epsilon_{\max }$ and $\Delta \mathrm{OD}(5.3 \mathrm{ps})=\Delta \mathrm{OD}_{\max }$, respectively, when neglecting the first maximum at about $50 \mathrm{fs}$ that is due to the internal conversion. Using Eq. (4) all transients from Fig. 2 were recalculated and transient spectra at different delays were obtained (see Fig. 5).

The calculation of the transient spectra was performed according to a modified Sulzer-Wieland ansatz using Eqs. (8) and (9) (see appendix). The absorption spectrum of CHT has two maxima in the UV (see Fig. 1) which are considered via $\epsilon(\nu, T)=\epsilon_{1}(\nu, T)+\epsilon_{2}(\nu, T)$. For the elucidation of transient spectra only the maximum at $261 \mathrm{~nm}$ could be resolved in the absorption spectrum (Fig. 1); the second transition has its maximum below $200 \mathrm{~nm}$ which could not be resolved with our spectrometer. Therefore, the asymmetry inserted by Eq. (9) was only considered for the transition at $261 \mathrm{~nm}\left(38314 \mathrm{~cm}^{-1}\right)$ while for the second transition no asymmetry was taken into account. This is justified because the spectral contribution of the second transition in the experimental accessible region between 300 and $400 \mathrm{~nm}$ is expected to be small. The parameters in Eq. (8) for CHT have been adapted from Ref. [23]; to account for the 


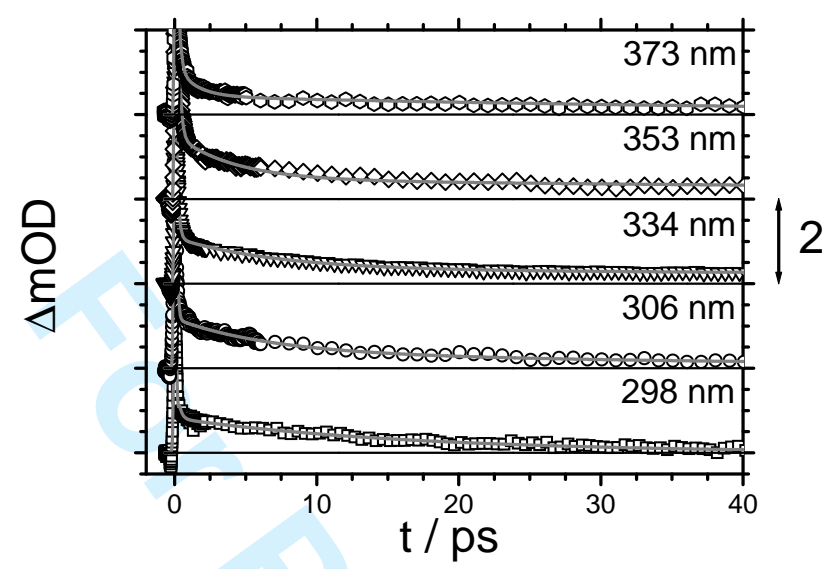

Figure 4: Pump-probe experiment of CHTd8 with $\lambda_{p u m p}=263 \mathrm{~nm}$ and $\lambda_{p r}$ between 298 and $373 \mathrm{~nm}$ highlighting the regime of vibrational relaxation. The length of the arrow corresponds to $2 \Delta \mathrm{mOD}$.

spectral red-shift in cyclohexane compared to gas phase data the parameter $\nu_{0}$ has been changed from 39300 (gas phase) to $38314 \mathrm{~cm}^{-1}$ (liquid phase: cyclohexane). The experimental data were evaluated according to Eq. (4) and are also shown for specific delay times. Fig. 5 clearly demonstrates that after 500 fs the temporal evolution of CHT can be understood in terms of vibrational relaxation that can be described by the modified Sulzer-Wieland formalism (see section 4 for details). At earlier times (hexagons in the inset of Fig. 5, representing a delay of $100 \mathrm{fs}$ ) no agreement between calculations and experimental results can be found. This indicates that another state rather than the "hot" ground state is observed and consequently must be assigned to spectral properties of the internal conversion. The absorption coefficient given at $100 \mathrm{fs}$ are only approximate values because of polarization effects.

According to our analysis, each delay time can be quantitatively simulated by a modified Sulzer-Wieland formalism that describes spectra at different temperatures. Therefore, one can assign each delay to a certain temperature. On the other hand, it is known (see, e.g., textbooks of statistical mechanics) that the temperature can be related to the vibrational energy $\mathrm{U}_{\text {vib }}(\mathrm{T})$ via

$$
U(T)=\sum_{i=1}^{n_{v}} \frac{R \Theta_{i}}{\exp \left\{\frac{\Theta_{i}}{T}\right\}-1}
$$

where $i$ denotes the different vibrational frequencies as given in Ref. [23] and $\Theta_{i}$ are the corresponding vibrational temperatures (see appendix). As a result, we obtain a relationship between delay time and $\mathrm{U}_{v i b}(\mathrm{~T})$ as displayed in Fig. 6. A simple monoexponential fit gives a decay time of $(8.5 \pm 1)$ ps which is assigned to the vibrational relaxation time of CHT in cyclohexane. This time constant is in reasonable agreement with investigations in other solvents like ethanol and 


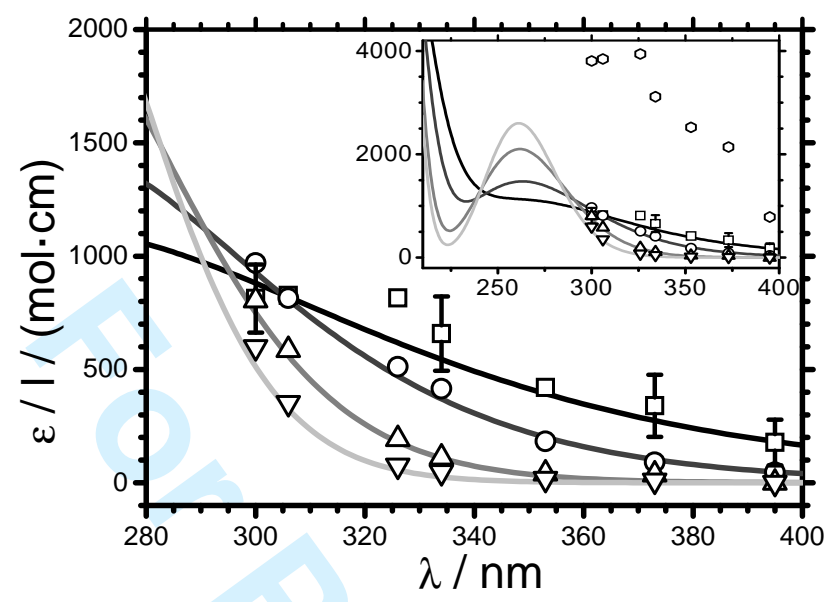

Figure 5: Transient spectra of CHT in cyclohexane, constructed with the method described in section 3. The symbols represent delay times of $\square: 500 \mathrm{fs}, \bigcirc: 5 \mathrm{ps}, \triangle: 20 \mathrm{ps}, \nabla: 90 \mathrm{ps}$. The curves are fits according to the modified Wieland-Sulzer ansatz at $\mathrm{T}=2200 \mathrm{~K}$ (black), $1200 \mathrm{~K}$ (dark gray), $500 \mathrm{~K}$ (gray) and $300 \mathrm{~K}$ (light gray). In the inset, the spectrum between 220 and $400 \mathrm{~nm}$ is shown. Added are the extinction coefficients at $\tau=100 \mathrm{fs}$ (hexagons).

heptane where relaxation times of $7.9 \mathrm{ps}$ and $9.5 \mathrm{ps}$ respectively were obtained by Benzler et. al. $[13,14]$ who used a slightly higher excitation energy $(41,000$ $\mathrm{cm}^{-1}$ compared to $38000 \mathrm{~cm}^{-1}$ in our studies).

\section{Discussion}

In most publications on pump-probe studies in liquid phase, the contribution of vibrational relaxation is fitted in terms of one exponential or the sum of several exponential decays. The decay constant for this process is thought to depend on the probe wavelength: the shorter the wavelength, the longer the time constant (for a more thorough explanation see, e.g., ref. [24]). The same effect can be observed for CHT: Fitting the data to a three-exponential decay function as described above, where the first accounts for the $\mathrm{H}$-shift and the last mostly describes a small offset (except for the measurements at 306 and $298 \mathrm{~nm}$ ) leads to results with small $\chi^{2}$-values. However, when considering the residuals of the transients of CHT after subtraction from the three-exponential fit (see Fig. 7) one observes a wavelength independent deviation from the exponential behavior indicating a general error when using this fitting function. These deviations can be explained by two factors:

(i) Instead of constructing transient spectra and comparing them to spectra obtained by the Sulzer-Wieland approach, it is possible to use a global fitting procedure to receive the time constant for vibrational cooling. The appropriate decay function is given by: 


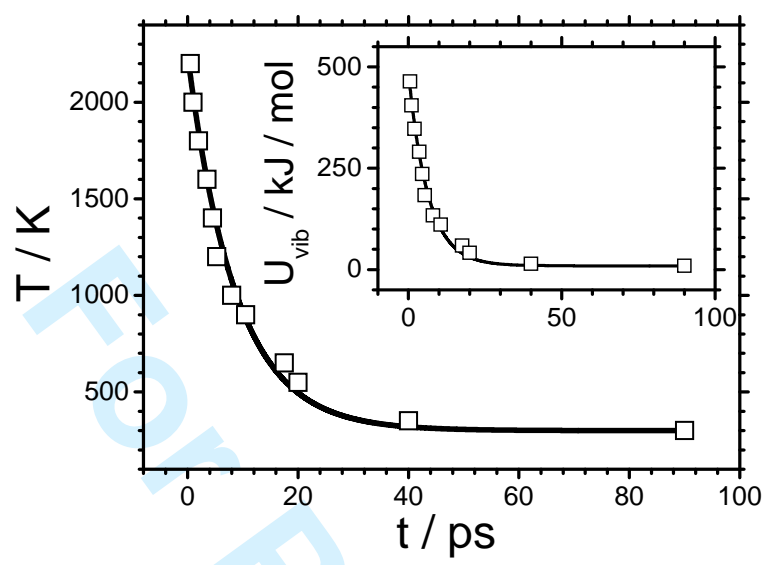

Figure 6: Time dependence of vibrational cooling of CHT in cyclohexane following excitation at $\lambda=263 \mathrm{~nm}$.

$$
\begin{aligned}
& f(t)=C_{1} \exp \left\{-t / \tau_{I C}\right\}+C_{2}[\epsilon(b, 300 \mathrm{~K}+ \\
& \left.\left.1900 \mathrm{~K} \exp \left\{-t / \tau_{v i b}\right\}\right)-\epsilon(b, 300 \mathrm{~K})\right]+C_{3},
\end{aligned}
$$

where $\epsilon$ is used as defined in Eq. (8) and $b=\left(\frac{\nu-\nu_{0}}{\Delta \nu_{0}}\right)^{2}$ (see appendix for a detailed explanation of the used parameters). The fitting parameters according to a Levenberg-Marquardt algorithm are $\Theta=420 \mathrm{~K}$ and $\tau_{v i b}=$ 10.3 ps which are in good argreement with $\Theta \approx 430 \mathrm{~K}$ as proposed by Hippler et al. [11] and used in the former analysis. The proposed decay according to Eq. (6) removes the wavelength independent structure of the residuals except the first extremum around $t=450 \mathrm{fs}$ (see (ii)). Although neither the time constant for vibrational relaxation nor the parameter $\Theta$ deviate markedly from the former analysis and is in accordance with former publications, the method should not be used without care: In most experiments, vibrational relaxation can only be analyzed by a decaying slope rather than by constructing a whole spectrum. Considering only the measurements for $\lambda \geq 326 \mathrm{~nm}$, the global fitting procedure delivers a time constant of $\tau_{v i b} \approx 23 \mathrm{ps}$ and $\Theta>1000 \mathrm{~K}$ since the region of a $\Delta$ OD-rise is obviously wrongly predicted, eventually due to the signal to noise ratio or because the Sulzer-Wieland ansatz is not good enough. Hence, these parameters do not allow to obtain the appropriate behavior for shorter wavelengths. As a consequence, no comparable quantitative analysis for the CHTd8-system can be given.

(ii) As noted above, the first maximum of the residuals around 450 fs does not vanish when using Eq. (6) instead of an exponential decay for wavelengths 
of $\lambda \geq 326 \mathrm{~nm}$. For 306 and $298 \mathrm{~nm}$, the residuals exibit negative values. This behavior can be explained as follows: According to Fig. 5, a higher $\Delta$ OD-value corresponds to a higher temperature for $\lambda \geq 326 \mathrm{~nm}$ while for 306 and $298 \mathrm{~nm}$ a higher temperature is indicated by a lower $\Delta \mathrm{OD}$-value. In consequence, the residuals indicate a higher initial temperature than the one obtained by the exponential fit. There are at least two possible reasons for this behavior:

(iia) During the first few hundreds femtoseconds the molecules are supposed to rotate freely as can be studied by, e.g., transient anisotropy (see Ref. [25] and references cited therein). Moreover, the average effective collision time of CHT in various solvents was found to be 2$2.5 \mathrm{ps}^{-1}$ by Benzler et. al. [14], which coincides with the delay time of the extremum. Therefore, one may conclude that the molecule remains its initial temperature until its first collision. The higher temperature is therefore due to non-statistical collision processes at short time delays.

(iib) The initial energy distribution of the molecules is not canonical. This might affect the probe process since no molecule has initially an internal energy that is below the threshold of being probed. Therefore, the experiment might overestimate the initial temperature.

As mentioned, collision induced vibrational cooling of CHT has already been investigated by Benzler et al. $[13,14]$ (see sections 1 and 3). The authors used pump-probe experiments with a time resolution of $500 \mathrm{fs}, \lambda_{\text {pump }}=248 \mathrm{~nm}$ 
and $\lambda_{\text {probe }}=225$ and $313 \mathrm{~nm}$. In order to extract the time dependence of the cooling, hot spectra from shock tube data [12] have been interpolated to higher temperatures. While this method seems to be an efficient way to extract the time dependence of collisional energy transfer, the presented results offer additional information:

(a) Transient spectra have been extracted and adopted to $\epsilon(t)$-values (mostly, the relative change in optical density is measured without absolute values).

(b) The time dependent spectra could be associated with a (vibrational) temperature by the modified Sulzer-Wieland ansatz. While this model includes several assumptions, it is sufficient to describe spectra within the uncertainties of the measurement. Other models based on spectral moments have been given [26]-[30]; however, the modified Sulzer-Wieland ansatz has the advantage of an analytic expression and a small set of adjustable parameters.

(c) For this analysis it is necessary to identify a (local) maximum in the $\triangle \mathrm{OD}(t)$-measurements at a certain probe wavelength - in the case of $\mathrm{CHT}$ at $\lambda_{\text {probe }}=298 \mathrm{~nm}$ that can be assigned to the cooling process. It is expected that such extrema should occur on a ps timescale in many systems and hence, should be far away from cross correlation contributions around time zero supposed the time resolution is high enough. In any case, this local extremum is fundamental for the construction of the $\epsilon(t)$ spectra. One of the main results of this analysis is that local extrema in ultrafast absorption spectroscopy do not necessarily have to be assigned to additional time constants and physical processes. As shown, vibrational cooling in the electronic ground state has also to be considered.

(d) A sufficient time resolution allows for the observation of non-statistical effects in an otherwise exponential temperature decay.

While the presented method gives a lot of information concerning vibrational cooling, there are as well several problems which can be seen in the case of CHTd8: Here, there neither exist parameters for the modified Sulzer-Wieland model (i.e., no temperature dependent spectra) nor a maximum in the $\Delta \mathrm{OD}(\mathrm{t})$ curve to extract transient spectra. Therefore, little information on a quantitative level for the vibrational cooling of this molecule can be obtained.

Finally, it is worth to briefly compare the presented experiments with the investigation of vibrational cooling in IR-pump Raman-probe studies (see Refs. [31][33] and references cited therein). In these kind of experiments, an IR-photon is used to excite a high frequency mode (normaly a $\mathrm{CH}$-strech-mode). Afterward, a narrow bandwidth VIS-pulse induces Raman scattering which allows to record the time dependent population of various vibrational modes. This powerful technique offers the possibility to study vibrational cooling as well as internal vibrational redistribution on a ps-timescale. However, the results presented so far only have a time resolution of $\sim 1 \mathrm{ps}$ and it is uncertain whether the dynamics 
following excitation of a single mode are able to explain the ground state dynamics following UV-irradiation and ultrafast internal conversions. The often used three stage model to describe Raman experiments [32]-[34] is a microcanonical picture, i.e., it keeps track of every vibrational mode. In order to describe highly excited molecules, especially those with many vibrational degrees of freedom, the introduction of a canonical picture (and hence a vibrational temperature as the only defining parameter of the system) seems adequate. This offers a simple model of the underlying dynamics.

\section{Conclusion}

In this work, we have performed pump-probe investigations of CHT and CHTd8 in cyclohexane with a time resolution of $50 \mathrm{fs}$ at probe wavelengths between 300 and $400 \mathrm{~nm}$ after excitation to the $S_{2}$-state with $\lambda_{\text {pump }}=263 \mathrm{~nm}$ in order to study vibrational cooling on an ultrafast timescale. After internal conversion within the first hundreds of fs, the molecule resides in the vibrationally excited electronic ground state. Using results of earlier works on microcanonical and canonical energy distributions, we could extract and simulate transient spectra of CHT by applying a modified Sulzer-Wieland equation. As a result, each delay time ( $\geq 500 \mathrm{fs}$ ) in the transient absorption profiles that monitors a microcanonical distribution can be interpreted in terms of a canonical distribution at a specific temperature and hence a vibrational energy. From this we obtained a time constant for the vibrational relaxation $\tau_{v i b}$ for $\mathrm{CHT}$ of $8.5 \mathrm{ps}$ in cyclohexane as solvent.

Acknowledgments This work has been financially supported by the Deutsche Forschungsgemeinschaft (DFG) and the University of Karlsruhe (TH).

\section{A. Sulzer-Wieland ansatz}

\section{A.1. Basic equations}

In the classical Sulzer-Wieland approach the upper state potential is assumed to be repulsive and without curvature. Moreover, the oscillator strength of a transition does not depend on the temperature such that the extinction coefficient can be expressed by $[15,35]$

$$
\frac{\epsilon(\nu, T)}{\nu} \stackrel{\propto}{\sim} \epsilon(\nu, T)=\epsilon_{0}^{\max } \sum_{n=0}^{\infty} N_{n}(T)\left|\Psi_{n}(\nu)\right|^{2}
$$

where $\epsilon_{0}^{\max }$ is the extinction coefficient at zero temperature, $\Psi_{n}(\nu)$ is the $n$-th vibrational wave function of the electronic ground state and $N_{n}(T)$ is its occupation number. In the Sulzer-Wieland model the ground state is described by a harmonic oscillator whose wave function is given by $\Psi_{n}^{\text {harm }}(\xi)=G_{n} e^{-\xi / 2} H_{n}(\xi)$, where $\xi=\left(\nu-\nu_{0}\right) / \Delta \nu_{0}, \nu_{0}$ is the mean transition energy [s $\left.{ }^{-1}\right], G_{n}$ the normalization constant and $H_{n}$ the $n$-th Hermite polynomial. The factor $\Delta \nu_{0}$ is 
given by the slope of the upper state potential. The occupation number for a harmonic potential is given by $N_{n}^{\text {harm }}=(1-z) z^{n}$, where $z=\exp \{-\Theta / T\}$ with $\Theta=\hbar \omega_{0} / k_{B}$. The factor $\nu$ on the left hand side of Eq. (7) is neglected. Then, the temperature dependence can be calculated to give

$$
\begin{aligned}
& \epsilon(\nu, T)=\epsilon_{0}^{\max }\left[\tanh \left(\frac{\Theta_{0}}{2 T}\right)\right]^{\frac{1}{2}} \times \\
& \exp \left\{-\tanh \left(\frac{\Theta_{0}}{2 T}\right)\left(\frac{\nu-\nu_{0}}{\Delta \nu_{0}}\right)^{2}\right\}
\end{aligned}
$$

Two extensions are given to account for a red-shift of the absorption spectra with increasing temperature and for an asymmetry of the recorded spectra $[12,11]$ :

1. In the first extension, Eq. 8 is multiplied by $\nu / \nu_{0}$ and the frequency $\nu_{0}$ is modified through the average vibrational energy of the $\omega_{0}$-vibration:

$$
\nu_{0, e f f}=\nu_{0}-\frac{\omega_{0}}{\exp \{\Theta / T\}-1}
$$

2. If more than one spectral change inducing mode is present, the SulzerWieland equation (8) can be modified by

$$
\epsilon(\nu, T)=\epsilon^{S W}(\nu, T) \frac{\nu}{\nu_{0}}\left(1+A \xi+B \xi^{3}\right),
$$

where $A$ and $B$ are parameters depending on $z, \omega_{0}$ and $\Delta \nu_{0}$ only (see Ref. [11]).

\section{A.2. Multidimensional Sulzer-Wieland ansatz}

The classical Sulzer-Wieland ansatz was originally developed for diatomic molecules exhibiting a single vibration that is treated in the approximation of a harmonic oscillator. However, a polyatomic molecule exhibits a density of vibrational states that is much different from a diatomic. The differences between the classical and a multidimensional model are shortly discussed in the following:

For a polyatomic molecule, the Sulzer-Wieland equation can be written as

$$
\epsilon_{\text {poly }}(\nu, T)=\epsilon_{0}^{\max } \sum_{i=1}^{n_{v}} \sum_{n=0}^{\infty} N_{n, i}(T)\left|\Psi_{n, i}(\nu)\right|^{2}
$$

where $i$ runs over all vibrations of the molecule and the occupation number is given by $N_{n, i}(T)=\exp \left\{-n \Theta_{i} / T\right\} /\left(\sum_{i, n} \exp \left\{-n \Theta_{i} / T\right\}\right)$. Here, $\Theta_{i}$ are the vibrational temperatures for the $i$-th vibrational mode. This model implies that all wavefunctions can be reflected at the same slope of the upper potential energy surface (idealized as a straight line). Fig. 8 shows plots at different temperatures where the vibrational frequencies of CHT were used to calculate $\epsilon_{\text {poly }}(\nu, T)$. These plots are compared with the results obtained via the one 


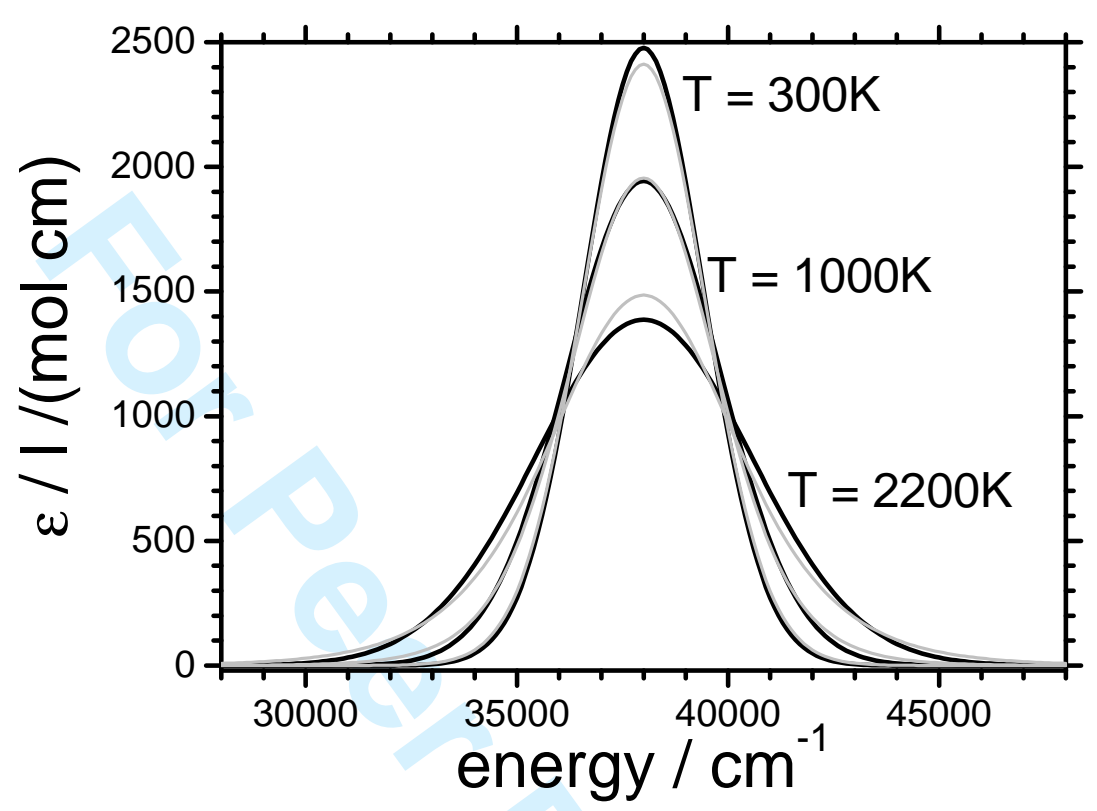

Figure 8: Comparison of the temperature dependency of the absorption spectra according to a one dimensional Sulzer-Wieland model (black curves) and the multidimensional treatment (gray curves) for different temperatures. The chosen parameters were $\nu_{0}=38,000 \mathrm{~cm}^{-1}$ and $\Delta \nu=2,000 \mathrm{~cm}^{-1}$. The $\Theta_{i}$ are the values for CHT (see [23]).

dimensional Sulzer-Wieland approach. As can be seen, the one dimensional formula is able to represent the multidimensional model for an adequate choice of $\Theta_{0}$ at least up to a temperature of $2200 \mathrm{~K}$. The shapes of the spectra are slightly different, nevertheless, it seems justified to work with a one-dimensional representation. 


\section{References}

[1] P. M. Borell, H.-G. Löhmannsröben, K. Luther, Chem. Phys. Lett. 136 (1987) 371.

[2] P. J. Reid, A. P. Shreve, R. A. Mathies, J. Phys. Chem. 97 (1993) 12691.

[3] P. J. Reid, M. K. Lawless, S. D. Wickham, R. A. Mathies, J. Phys. Chem. 98 (1994) 5597.

[4] M. K. Lawless, S. D. Wickham, R. A. Mathies, Acc. Chem. Res. 28 (1995) 493.

[5] A. Hertwig, H. Hippler, H. Schmid, A. N. Unterreiner, Phys. Chem. Chem. Phys. 1 (1999) 5129.

[6] S. A. Trushin, S. Diemer, W. Fuß, K. L. Kompa,W. E. Schmid, Phys. Chem. Chem. Phys. 1 (1999) 1431.

[7] H.-M. Steuhl, C. Bornemann, M. Klessinger, J. Chem. Eur. 5 (1999) 2404.

[8] C. Y. Ruan, V. A. Lobastov, R. Srinivasan, B. M. Goodson, H. Ihee, A. H. Zewail, PNAS 98 (2001) 7117.

[9] H. Hippler, M. Olzmann, O. Schalk, A. N. Unterreiner, Z. Phys. Chem. 219 (2005) 389.

[10] O. Schalk, A.-N. Unterreiner, J. Phys. Chem. A 111 (2007) 3231.

[11] H. Hippler, K. Luther, J. Troe, H. J. Wendelken, J. Phys. Chem. 79 (1983) 239.

[12] D. C. Astholz, L. Brouwer, J. Troe, Ber. Bunsenges. Phys. Chem. 85 (1981) 559 .

[13] J. Benzler, S. Linkersdörfer, K. Luther, Ber. Bunsenges. Phys. Chem. 100 (1996) 1252.

[14] J. Benzler, S. Linkersdörfer, K. Luther, J. Phys. Chem. 106 (1997) 4992.

[15] P. Sulzer, K. Wieland, Helv. Phys. Acta 85 (1952) 559.

[16] C. Engdahl, G. Jonsäll, P. Ahlberg, J. Am. Chem. Soc. 105 (1083) 891.

[17] M. Lenzner, C. Spielmann, E. Winter, F. Krausz, A. J. Schmidt, Opt. Lett. 20 (1995) 1397.

[18] S. Sartania, Z. Cheng, M. Lenzner, G. Tempea, C. Spielmann, F. Krausz, K. Ferencz, Opt. Lett. 22 (1997) 1562.

[19] A.-N. Unterreiner, PhD thesis, Universität Karlsruhe (TH), 1998, ISBN: 3-89653-368-1. 
[20] T. Wilhelm, J. Piel, E. Riedle, Opt. Lett. 22 (1997) 1494.

[21] J. Piel, M. Beutter, E. Riedle, Opt. Lett. 25 (2000) 180.

[22] R. P. Frueholz, R. Rianda, A. Kuppermann, Chem. Phys. Lett. 55 (1978) 88.

[23] D. C. Astholz, J. Troe, W. Wieters, J. Chem. Phys. 70 (1979) 5107.

[24] O. Schalk, H. Brands, T. S. Balaban, A.-N. Unterreiner, J. Phys. Chem. A 112 (2008) 1719.

[25] O. Schalk, A.-N. Unterreiner, J. Chem. Phys. (submitted).

[26] E. A. Gislason, J. Chem. Phys. 58 (1973) 3702.

[27] S. M. Adler-Golden, Chem. Phys. 64 (1982) 421.

[28] J. A. Joens, Chem. Phys. Lett. 138 (1987) 512.

[29] J. A. Joens, J. Phys. Chem. 97 (1993) 2527.

[30] R. D. Coalson, M. Karplus, J. Chem. Phys. 81 (1984) 2891.

[31] S. Shigeto, D. D. Dlott, Chem. Phys. Lett. 447 (2007) 134.

[32] S. Shigeto, Y. Pang, Y. Fang, D. D. Dlott, J. Phys. Chem. B 112 (2008) 232.

[33] N.-H. Seong, Y. Fang, D. D. Dlott, J. Phys. Chem. A 113 (2009) 1445.

[34] A. Nitzan, J. Jortner, Mol. Phys. 25 (1973) 713.

[35] G. Herzberg, Molecular Spectra of Molecular Structure, Vol. 3, Van Nostrand Reinhold, New York (1966). 


\section{List of Figures}

1 UV-Vis spectra of CHT and CHTd8 in cyclohexane, adopted from Ref. [9]. . . . . . . . . . . . . . . . . . .

2 Pump-probe experiment of CHT with $\lambda_{\text {pump }}=263 \mathrm{~nm}$ and $\lambda_{p r}$ between 298 and $373 \mathrm{~nm}$ highlighting the regime of vibrational relaxation. The length of the arrow corresponds to $2 \Delta$ mOD. . . 5

3 Pump-probe experiment of CHT with $\lambda_{p u m p}=263 \mathrm{~nm}$ and $\lambda_{p r}=$ $298 \mathrm{~nm}$. The inset shows the response on a longer timescale. . . 6

4 Pump-probe experiment of CHTd8 with $\lambda_{\text {pump }}=263 \mathrm{~nm}$ and $\lambda_{p r}$ between 298 and $373 \mathrm{~nm}$ highlighting the regime of vibrational relaxation. The length of the arrow corresponds to $2 \Delta \mathrm{mOD}$. . .

5 Transient spectra of CHT in cyclohexane, constructed with the method described in section 3 . The symbols represent delay times of $\square: 500 \mathrm{fs}, \bigcirc: 5 \mathrm{ps}, \triangle: 20 \mathrm{ps}, \nabla: 90 \mathrm{ps}$. The curves are fits according to the modified Wieland-Sulzer ansatz at $\mathrm{T}=2200 \mathrm{~K}$ (black), $1200 \mathrm{~K}$ (dark gray), $500 \mathrm{~K}$ (gray) and $300 \mathrm{~K}$ (light gray). In the inset, the spectrum between 220 and $400 \mathrm{~nm}$ is shown. Added are the extinction coefficients at $\tau=100 \mathrm{fs}$ (hexagons).

6 Time dependence of vibrational cooling of CHT in cyclohexane following excitation at $\lambda=263 \mathrm{~nm} . \ldots \ldots$. . . . . .

7 Residuals of the transients of CHT after subtraction from a threeexponential fit for a pump wavelength of $263 \mathrm{~nm}$ and various probe wavelength $(298,326,334,353$ and $373 \mathrm{~nm})$. In the inset, the average over the residuals of the four longest wavelengths are shown. See text for discussion. . . . . . . . . . . .

8 Comparison of the temperature dependency of the absorption spectra according to a one dimensional Sulzer-Wieland model (black curves) and the multidimensional treatment (gray curves) for different temperatures. The chosen parameters were $\nu_{0}=$ $38,000 \mathrm{~cm}^{-1}$ and $\Delta \nu=2,000 \mathrm{~cm}^{-1}$. The $\Theta_{i}$ are the values for CHT (see [23]). 